\title{
Novel Classification Algorithm for Ballistic Target based on HRRP frame
}

\author{
A.R. Persico, Student Member, IEEE and C.V. Ilioudis, Student Member, IEEE \\ and C. Clemente, Member, IEEE, and J. J. Soraghan, Senior Member, IEEE
}

\begin{abstract}
Nowadays the identification of ballistic missile warheads in a cloud of decoys and debris is essential for defence systems in order to optimize the use of ammunition resources, avoiding to run out of all the available interceptors in vain. This paper introduces a novel solution for the classification of ballistic targets based on the computation of the inverse Radon transform of the target signatures, represented by a high resolution range profile frame acquired within an entire period of the main rotation of the target. Namely, the precession for warheads and the tumbling for decoys are taken into account. Following, the pseudo-Zernike moments of the resulting transformation are evaluated as the final feature vector for the classifier. The extracted features guarantee robustness against target's dimensions and rotation velocity, and the initial phase of the target's motion. The classification results on simulated data are shown for different polarizations of the electromagnetic radar waveform and for various operational conditions, confirming the validity of the algorithm.
\end{abstract}

Keywords-Ballistic Missile Defence, High Resolution Range Profile, Inverse Radon Transform, Pseudo-Zernike Moments, Ballistic Target Classification

\section{INTRODUCTION}

Since the early stages of the development of InterContinental-range Ballistic Missiles (ICBMs) and Submarine-Launched Ballistic Missiles (SLBMs), many countries invest annually a significant budget into research and production of countermeasures in order to minimize the effectiveness of Ballistic Missile Defence (BMD) systems [1]. One of the most common practices is the use of a large number of decoys, or false targets, wich aim to confuse the defence systems. Currently, different decoy strategies are available, such as replica decoys, decoys using signature diversity and decoys using antisimulation [2]. Specifically, the lightweight decoys are a very attractive option against exo-atmospheric defences, as the missile's warhead size and range depend on the

A.R. Persico, C.V. Ilioudis, C. Clemente and J. J. Soraghan are with University of Strathclyde, Centre for Signal and Image Processing, EEE, 204, George Street, G1 1XW, Glasgow, UK. E-mail: adriano.rosario.persico@gmail.com, c.ilioudis@strath.ac.uk, carmine.clemente@strath.ac.uk,j.soraghan@strath.ac.uk. weight of the total carried payload. Hence, missiles can be equipped with a large number of lightweight decoys without affecting the maximum warhead range [1].

Long-range BM move on sub-orbital trajectories and their ranges typically depend on the altitude achieved by using one ore more boosters. The longer part of a BM flight takes place in the exo-atmosphere and it is commonly known as the mid-course phase. During this phase, the lightweight decoys are released as both the decoys and the much heavier warhead travel on similar trajectories due to the absence of atmospheric drag in the vacuum of space [3]. In addition to the intentional decoys, missiles also release incidental debris and deployment hardware, e.g. boosters for missile launch, which can pose an additional source of interference on radar returns. In absence of reliable target identification, the defence system has to intercept all the detected targets, including decoys, in order to prevent the warhead from reaching its aim. Since the anti-ballistic missile systems have a limited number of interceptors, the challenge of Ballistic Targets (BTs) classification, identifying the warhead into a cloud of decoys and debris, is of fundamental importance. Specifically, the development of classification algorithms with high level of efficiency, low computational cost and short time decision is desirable not only for ground-based and sea-based defence station, but even for the On-Board Computer carried by the interceptor. The main reason for such a need is that the defence system may have to launch its interceptors before the lightweight decoys could be discriminated in order to intercept threats very far away from the interceptor deployment site [1]. Moreover, once the warhead has been identified, it is essential for the seeker on the interceptor to determine the aim point on the Re-enter Vehicle (RV) for terminal guidance and effective impact during engagement [4].

It is very important to note that a defence system's efficiency can be critically affected by decoys in two related ways. Specifically, if a decoy is classified as a warhead (false alarm), the defence may run out its ammunition of interceptors prematurely. By contrast, the misclassification of a warhead (leakage) may lead to 
catastrophic consequences [2]. A window of opportunity to discriminate between warheads and lightweight decoys occurs during the re-enter phase, since decoys would slow down more rapidly due to the atmospheric drag than the warhead. However, the target identification in this phase may be not useful for interception due its short duration (few seconds), and because the warhead could have already passed the minimum intercept altitude for an above-the-atmosphere interceptor [2]. For such reasons, the mid-course phase usually represents the most useful flight part for intercepting missiles due to its relatively long duration and the absence of tactical manoeuvring of targets as they are in free-flight motion. The capability to distinguish between warheads and decoys during the mid-course phase is a topic widely investigated in the literature with the developed target identification algorithms being mainly based on the different micro-motions exhibited by BTs. Specifically, the warheads are typically spin-stabilized to ensure that they do not deviate from the intended ballistic trajectories, while also exhibiting precession and nutation motion as effect of the Earth's gravity [4]. By contrast, decoys tumble when released by the missiles due to the gravity and the absence of a spinning motor [5], [6].

Information regarding target's micro-motions can be extracted from both Doppler and range analysis of the radar returns. The effect in the Doppler domain has been firstly described by V. Chen in [7], and it is well known as micro-Doppler effect. The authors in [3] describe an adaptive framework for BM classification, demonstrating the capability to discriminate between warheads and decoys using the micro-Doppler information. In particular, this framework is based on the evaluation of the spectrogram and the Cadence Velocity Diagram (CVD), which allows to extract the cadence of the micro-Doppler frequencies within the received echo. In order to perform classification, the CVD is used as the target's signature from which a feature vector is extracted by using several approaches, which are different in terms of computational cost and feature vector dimension. On the other hand, in range analysis the micro-motions exhibited by the target lead to range migrations of its principal scattering points which are observable through a High Resolution Range Profile (HRRP) frame obtained by a wideband radar. In particular, the use of Stepped Frequency Waveforms (SFWs) for achieving a HRRP in a BMD scenario is thoroughly discussed in [8], highlighting the distortion introduced by target's micro-motions. In the last decades the Frequency Stepped Chirp Radars (FSCR) have found application in missile terminal guidance [9]. The authors in [9] proposed a novel algorithm for the velocity estimation for missile-borne FSCR, with the aim to compensate the distortion in the HRRP due to relative motion between the radar and the target. Specifically, the algorithm is based on the evaluation of the waveform entropy in the Doppler amplitude spectrum. The authors in [6] investigated the effect of target micro-motions on the space distribution of target principal scatterers in the HRRP over the time. In particular, an analysis on the capability to discriminate between different target shapes and micro-motions (such as precession, wobbling and tumbling) is conducted by a graphical analysis which combines information extracted from the HRRP frame and a Time-Frequency Distribution (TFD).

Many authors propose the use of the Radon Transform (RT) and its inverse transform for extracting information from target motion for imaging and classification purposes. Introduced in 1917 by Johann Radon, the RT is widely used in computer imaging applications, e.g. tomography [10]. The RT of a two-dimensional (2-D) function for a fixed angle is defined as the function projection (line integral) onto the line defined in the 2-D plane by that angle. In [11] and [12] the RT is used in order to detect linear FM signals. Since a line structure in the TF plane is projected onto a point in the Radon transform the chirp rate value of a linear FM signal can be estimated evaluating the concentration of the Wigner distribution projection along different directions (angles). Since the effect of a rotating scatterer in the rangeslow time domain is equivalent to the RT of its space distribution function, the Inverse Radon Transform (IRT) is proposed in the literature to reconstruct a 2-D image of the target as a back-projection approach. Specifically in [13] two IRT based methods are presented for image reconstruction of rotating parts of a target such as airplane or helicopter rotors. The first method uses the real-valued IRT of the echoes modulus, while the second one applies the complex-valued IRT to the complex echoes guaranteeing higher image resolution by performing a coherent integration. Additionally, the IRT is also proposed in the (Inverse) Synthetic Aperture Radar (ISAR/SAR) processing in order to separate the echoes occurred by the target's rigid body from those generated by its rotating parts. In fact, when the frequency modulations due to the moving parts of targets are not filtered out, the micro-Doppler effect introduces a distortion in the SAR/ISAR images. In order to address this issue, in [14] two techniques are proposed. The first technique is based on the TFD analysis of radar returns. Specifically, the spectrogram of the echo is evaluated for various window sizes, since the contribution of rotating parts leads to a high concentration in the narrow-window spectrogram, while the rigid body contribution produces a high concentration into wide-window spectrogram. The 
second approach is based on the IRT computation of the TFD of the received echo. The authors in [15] describe a new approach for cleaning the ISAR image of a target from its rotating parts by applying the IRT on a frame of target range-profiles. The rotation period is firstly estimated, then from the IRT of the range profile frame the contribution of rotating parts is detected and filtered out. In [16] a novel technique for the extraction of precession parameters of a conical target is presented. In particular, the proposed algorithm is based on the Doppler analysis of the radar echo: the precession parameter (angle and rate) are estimated by analysing the spread of echo spectrum and the echo autocorrelation. Finally a 2-D image of target is reconstructed by applying the IRT on the echo TFD.

In this paper the results and findings on an Automatic Target Recognition (ATR) algorithm proposed in [17] are presented, with the aim to classify targets in a BMD scenario from a sequence of HRRPs. The algorithm is based on the IRT of the HRRP frame, which leads to a 2-D target signature containing information on target motions and the space distribution of its principal scattering points. Then from the target's signature a feature vector is extracted, whose elements are the pseudo-Zernike (pZ) moments extracted from the 2-D target signature. The $\mathrm{pZ}$ moments are very attractive for image classification due to their useful properties, such as scale, translation and rotation invariance [18]. For this specific classification approach the rotation invariance is fundamental to ensure robustness with respect to the initial phase of the target micro-motion. In this paper, the work presented in [17] is extended by introducing additional models for the radar return, investigating the effect of the microDoppler modulation and different acquisition scenario such as polarization diversity. Moreover, the algorithm is tested in the case of partial data frame available for the extraction of the target's signature.

The remainder of the paper is organized as follows. Section II introduces the target model for BM warheads and decoys. Section III describes the classification framework proposed in [17], highlighting in details how the microDoppler effect due to target micro-motion affects the target signature. In Section IV the effectiveness of the proposed algorithm is demonstrated and the performance analysis obtained from simulated data is shown. Section $\mathrm{V}$ shows the performance analysis of the framework when partial data are used for extracting the target signature. Section VI concludes the paper. The appendix contains the mathematical expressions of complex scattering coefficients for the considered target model.

\section{Radar High Resolution Range Profile FROM BTS}

In radar surveillance applications the SFWs are generally used in order to achieve the HRRP of a target by increasing the system's bandwidth. In this section the signal model for rotationally symmetric BTs is presented, analysing the effect of different target micro-motions on a HRRP frame.

\section{A. SFWs Radar based HRRP}

The SFWs comprises a sequence of $N$ narrowband sub-pulses, known as burst, which are integrated coherently into a single wideband signal. The carrier frequency of each sub-pulse increases pulse by pulse.

Let us consider the transmission of $M$ bursts with a fixed Pulse Repetition Frequency (PRF), the transmitted signal can be written as

$$
s_{t x}(t)=\sum_{m=0}^{M-1} \sum_{n=0}^{N-1} p(t-n T-m N T) e^{j 2 \pi f_{n} t}
$$

where $T$ is the Pulse Repetition Interval (PRI), $f_{n}$ the carrier frequency of the $n$-th sub-pulse, and where

$$
p(t)=\left\{\begin{array}{cc}
A e^{j \pi \frac{\Delta f}{\tau} t^{2}} & 0<t<\tau \\
0 & \text { otherwise }
\end{array}\right.
$$

with $A, \tau$ and $\Delta f$ being amplitude, time duration and bandwidth of the waveform sub-pulse, respectively. For a full-band SFW, $\Delta f$ is equal to the frequency step of the sub-carrier, such that $f_{n}=f_{c}+n \Delta f$, with $n=0, \cdots, N-1$, and where $f_{c}$ is the fundamental carrier frequency. Without loss of generality, in the following analysis a system using a full-band SFW is taken into consideration and $A$ is set equal to 1 .

The received echo from a target at the radio frequency is expressed as the superimposition of the signals from each principal scattering point. After the de-chirping operation, during which the received signal is mixed with reference signal, the compensation of the Residual Video Phase (RVP) and the sideling term of the echo envelope (as explained in details in [19]), the received sample corresponding to $n$-th sub-pulse of $m$-th burst is given by:

$$
s(n, m)=\sum_{i=1}^{N_{P}} \sigma_{i} e^{j \rho_{i}} e^{-j \frac{4 \pi}{c} f_{n} \Delta R}+w(n, m)
$$

with $m=0, \cdots, M-1$ and $n=0, \cdots, N-1, \sigma_{i}$ and $\rho_{i}$ are the modulus and the phase of the electromagnetic contribution of the $i$-th scattering point, and $\Delta R=$ $R_{M C}-R_{0}$, with $R_{M C}$ the distance between the radar and the centre of mass of the target and $R_{0}$ the reference 
range, and where $w(n, m)$ is a Additive White Gaussian Noise (AWGN) sample. It is worth noting that the phase of each scattering coefficient contains information about the distance of scatterer with respect to the centre of mass along the LOS.

The conventional method for extracting the HRRPs from the echoes of each transmitted bursts is based on the computation of the Inverse Discrete Fourier Transform (IDFT) along the stepped frequencies [19]. Specifically, in this paper the HRRP is defined as the square magnitude of IDFT of received signal samples, such that the $(\varepsilon, m)$-th element of the HRRP frame $\chi$ can be written as

$$
\chi(\varepsilon, m)=\left|\frac{1}{N} \sum_{n=0}^{N-1} \varpi(n) s(n, m) e^{j \frac{2 \pi n}{N} \varepsilon}\right|^{2}
$$

with $\varepsilon=1, \ldots, N$ and $m=1, \ldots, M$, and where $\varpi(\cdot)$ is the smoothing window.

\section{B. BTs micro-motion model}

During the flight onto the exo-atmospheric part of their sub-orbits, the missile warheads exhibit precession and nutation motions as represented in Figure 1a. In particular, the precession is composed by two micro-motions: the spinning of the target around its symmetry axis, and the conical movement, such that the symmetry axis rotates conically around the precession axis. The nutation is an oscillation of the symmetry axis perpendicular with respect to the precession axis. When the missile releases lightweight decoys, they starts to tumble due to the Earth gravity, as shown in Figure $1 \mathrm{~b}$.

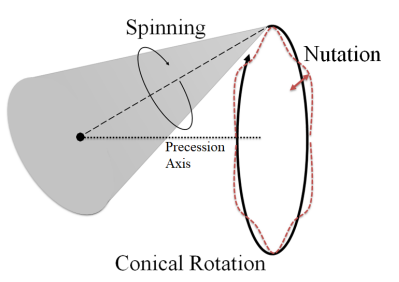

(a)

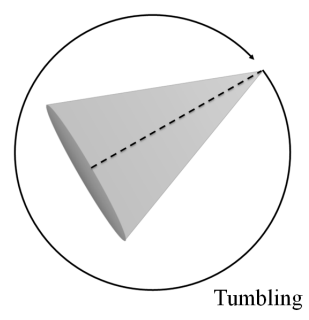

(b)
Figure 1: Ballistic target micro-motions: (a) warhead; (b) decoy.

Let us consider the coordinate system $(\hat{X}, \hat{Y}, \hat{Z})$ with origin in the centre of mass of the target, such that the $\hat{Z}$-axis is direct along the angular velocity vector of the conical rotation (hence along the precession axis), $\boldsymbol{w}_{r}$, and the position vector of radar lays on plane $\hat{X} \hat{Z}$, as shown in Figure 2. The unit vector $\boldsymbol{n}$ which represents

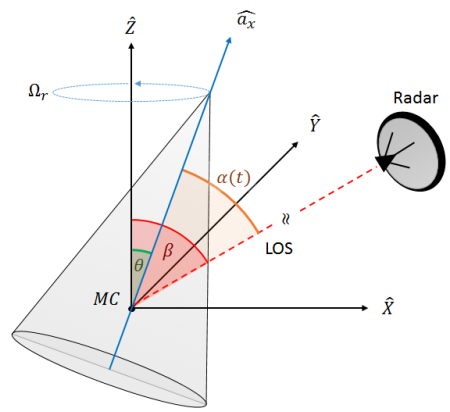

Figure 2: Coordinate system $(\hat{X}, \hat{Y}, \hat{Z})$

the direction of Line Of Sight (LOS) therefore expressed as:

$$
\boldsymbol{n}=[\sin (\beta), 0, \cos (\beta)]^{T}
$$

with $\beta$ being the position angle with respect to the $\hat{Z}$ axis.

It is worth noting that for a rotationally symmetric target the spinning does not change the radar view of the target. Moreover, in this paper the nutation is not taken into consideration for simplicity, since this oscillation movement does not produce significant range migration of the principal scatterers in the HRRP. Therefore, the unit vector $\boldsymbol{a}_{x}$, which identifies the direction of the target's axis of symmetry, varies on time due the conical rotation of the precession motion as follows:

$$
\begin{aligned}
& \widehat{\boldsymbol{a}_{x}}(t) \\
& =\left[\begin{array}{ccc}
\cos \left(\Omega_{r} t+\phi\right) & -\sin \left(\Omega_{r} t+\phi\right) & 0 \\
\sin \left(\Omega_{r} t+\phi\right) & \cos \left(\Omega_{r} t+\phi\right) & 0 \\
0 & 0 & 1
\end{array}\right]\left[\begin{array}{c}
\sin (\theta) \\
0 \\
\cos (\theta)
\end{array}\right] \\
& =\left[\begin{array}{c}
\cos \left(\Omega_{r} t+\phi\right) \sin (\theta) \\
\sin \left(\Omega_{r} t+\phi\right) \sin (\theta) \\
\cos (\theta)
\end{array}\right]
\end{aligned}
$$

where $\Omega_{r}=\left\|w_{r}\right\|$ is the precession angular velocity, $\phi$ is the initial phase of rotation and $\theta$ is the precession angle. The aspect angle $\alpha=\alpha(t)$, defined as the angle between the LOS and the symmetric axis, is given by:

$$
\begin{aligned}
& \alpha(t)=\cos ^{-1}\left(\boldsymbol{n} \cdot \widehat{\boldsymbol{a}_{x}}(t)\right)= \\
& \cos ^{-1}\left\{\sin \beta \sin \theta \cos \left(\Omega_{r} t+\phi\right)+\cos \beta \cos \theta\right\}
\end{aligned}
$$

The tumbling of decoys is defined as a rotation of the target such that the axis of symmetry is perpendicular to the rotation angular velocity vector. Hence, the aspect angle for decoys can be obtained from (7) for $\theta=90^{\circ}$, as follows

$$
\alpha(t)=\cos ^{-1}\left\{\sin (\beta) \cos \left(\Omega_{r} t+\phi\right)\right\}
$$


In the following analysis both $\beta$ and $\theta$ are assumed being constant during the observation time so that the variations of the aspect angle are consequence only of the described target's micro motions.

\section{Radar Cross Section Model}

According to the theory of diffraction at high frequency (short wavelength), the signal scattered by a target may be approximated by the sum of localized sources, represented by the principal scattering points on the object. Specifically the Radar Cross Section (RCS) of the target can be written as

$$
\sigma^{2}(f, \alpha)=\left|\sum_{i=1}^{N_{p}} \sigma_{i}(f, \alpha) e^{j \rho_{i}(f, \alpha)}\right|^{2}
$$

where $N_{p}$ is the total number of scatterers and $\sigma_{i}(\cdot) e^{j \rho_{i}(\cdot)}$ is the complex scattering coefficient of the $i$-th local source, which depends on both the carrier frequency $f$ and the aspect angle $\alpha$. The phase of the scattered field is [20]:

$$
\rho(f, \alpha)=\tan ^{-1}\left(\frac{\sum_{i=1}^{N_{p}} \sigma_{i}(f, \alpha) \sin \left(\rho_{i}(f, \alpha)\right)}{\sum_{i=1}^{P} \sigma_{i}(f, \alpha) \cos \left(\rho_{i}(f, \alpha)\right)}\right)
$$

The number of scattering points depends on the target shape. Let us consider the local coordinate system $(\hat{x}, \hat{y}, \hat{z})$, defined so that the $\hat{z}$-axis coincides with the symmetry axis of the target, $\widehat{a_{x}}$, and the LOS belongs to the plane $\hat{x} \hat{z}$ :

$$
\hat{x}=\hat{y} \times \hat{z} ; \quad \hat{y}=\widehat{a_{x}} \times \boldsymbol{n} ; \quad \hat{z} \equiv \widehat{a_{x}} ;
$$

The scattering points of the targets are located on the incident plane $\hat{x} \hat{z}$.

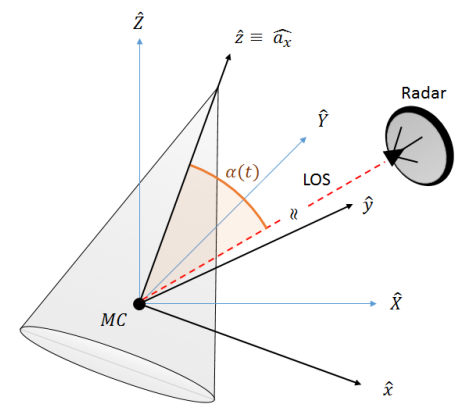

Figure 3: Local coordinate system $(\hat{x}, \hat{y}, \hat{z})$

In this paper three target shapes are considered: cone, cylinder and cone plus cylinder (see Figure 4). For a conical target three principal scattering points are considered: the first is in correspondence of the cone tip; the other two points are located on the intersection between the circumference at cone bottom and the incident plane $(\hat{x} \hat{z})$ (see Figure 4a). The cylindrical target is represented by four principal scattering points, two for each base, taken by intersecting the circumferences at the bases and the incident plane (see Figure 4b). Finally, for a target composed by a cone and a cylinder which share the base, five scattering points are considered. One represents the tip of the cone, while the other four are taken on the circumferences in correspondence of the cylinder bases on the incident plane (see Figure 4c).

In this work three mathematical models are considered for the complex coefficients of the target scattering points. The first is the Binary Scattering Coefficient (BSC) model, according to which the singular scattering properties of each scatterer are not taken into account for simplicity, considering the modulus of scattering coefficients as a binary function whose possible values are 0,1 . Specifically, this function represents a mask which depends on the aspect angle $\alpha(t)$, such that its value is 1 when there is a LOS for the scattering points, and 0 otherwise.

Let us consider the possible variation of $\alpha(t)$ into interval $[0, \pi]$. For the cone, $\sigma_{i}$ is 0 for the scattering point $P_{1}$ (see Figure 4a) when $\alpha(t) \in[\pi-\gamma, \pi]$ and for $P_{3}$ when $\alpha(t) \in[\gamma, \pi / 2]$, with $\gamma$ being the semi-angle of the cone; while for $P_{2}$ the occlusion never occurs for $\alpha(t) \in[0, \pi]$, e.g. $\sigma_{2}=1$ with $\alpha(t) \in[0, \pi]$. The values of the coefficients modulus in different aspect angles for the cone scatterers are synthesized in Table I.

Table II shows the coefficients modulus for the cylinder scatterers for different aspect angles. Specifically, $\sigma_{i}=0$ for $P_{1}$ when $\alpha(t)=\pi$; for $P_{2}$ when $\alpha(t)=0$; for $P_{3}$ when $\alpha(t) \in[0, \pi / 2]$ and for $P_{4}$ when $\alpha(t) \in[\pi / 2, \pi]$. Finally, for the cone plus cylinder, $\sigma_{i}=0$ for $P_{1}$ when $\alpha(t) \in[\pi-\gamma, \pi]$; for $P_{2}$ with $\alpha(t)=\pi$; for $P_{3}$ when $\alpha(t)=0$; for $P_{4}$ when $\alpha(t) \in[0, \pi / 2]$; for $P_{5}$ when $\alpha(t) \in[\gamma, \pi]$. Table III synthesizes how the coefficients modulus for the cone plus cylinder vary on the aspect angle.

Table I: Modulus of the scattering coefficients for the three principal scattering points $P_{1}, P_{2}$, and $P_{3}$ of the cone, with respect to the aspect angles $\alpha$.

\begin{tabular}{|c|c|c|c|}
\hline & $\sigma_{1}(\alpha)$ & $\sigma_{2}(\alpha)$ & $\sigma_{3}(\alpha)$ \\
\hline$\alpha<\gamma$ & 1 & 1 & 1 \\
\hline$\gamma \leq \alpha<\frac{\pi}{2}-\gamma$ & 1 & 1 & 0 \\
\hline$\frac{\pi}{2}-\gamma \leq \alpha<\frac{\pi}{2}$ & 1 & 1 & 0 \\
\hline$\frac{\pi}{2} \leq \alpha<\pi-\gamma$ & 1 & 1 & 1 \\
\hline$\pi-\gamma \leq \alpha \leq \pi$ & 0 & 1 & 1 \\
\hline
\end{tabular}

The phase of each coefficient depends on the relative distance between the centre of mass and the scattering 


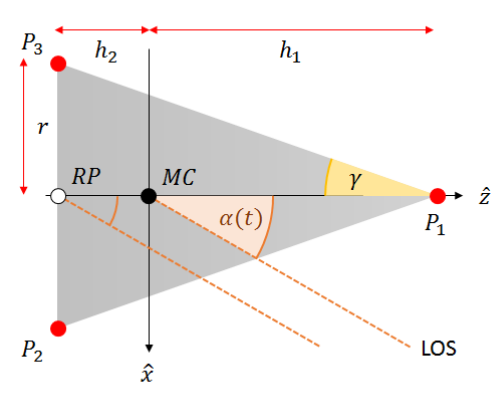

(a)

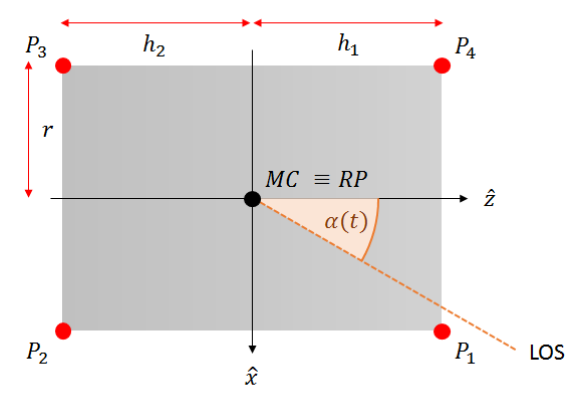

(b)

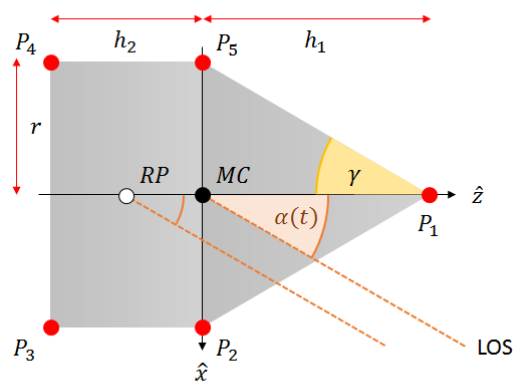

(c)

Figure 4: Target shape model: (a) Cone; (b) Cylinder; (c) Cone plus Cylinder.

Table II: Modulus of the scattering coefficients for the four principal scattering points $P_{1}, P_{2}, P_{3}$ and $P_{4}$ of the cylinder, with respect to the aspect angles $\alpha$.

\begin{tabular}{|c|c|c|c|c|}
\hline & $\sigma_{1}(\alpha)$ & $\sigma_{2}(\alpha)$ & $\sigma_{3}(\alpha)$ & $\sigma_{4}(\alpha)$ \\
\hline$\alpha=0$ & 1 & 0 & 0 & 1 \\
\hline $0<\alpha<\frac{\pi}{2}$ & 1 & 1 & 0 & 1 \\
\hline$\alpha=\frac{\pi}{2}$ & 1 & 1 & 0 & 0 \\
\hline$\frac{\pi}{2}<\alpha<\pi$ & 1 & 1 & 1 & 0 \\
\hline$\alpha=\pi$ & 0 & 1 & 1 & 0 \\
\hline
\end{tabular}

Table III: Modulus of the scattering coefficients for the four principal scattering points $P_{1}, P_{2}, P_{3}, P_{4}$ and $P_{5}$ of the cone plus cylinder, with respect to the aspect angles $\alpha$.

\begin{tabular}{|c|c|c|c|c|c|}
\hline & $\sigma_{1}(\alpha)$ & $\sigma_{2}(\alpha)$ & $\sigma_{3}(\alpha)$ & $\sigma_{4}(\alpha)$ & $\sigma_{5}(\alpha)$ \\
\hline$\alpha=0$ & 1 & 1 & 0 & 0 & 1 \\
\hline $0<\alpha<\gamma$ & 1 & 1 & 1 & 0 & 1 \\
\hline$\gamma \leq \alpha<\frac{\pi}{2}$ & 1 & 1 & 1 & 0 & 0 \\
\hline$\alpha=\frac{\pi}{2}$ & 1 & 1 & 1 & 0 & 0 \\
\hline$\frac{\pi}{2}<\alpha<\pi-\gamma$ & 1 & 1 & 1 & 1 & 0 \\
\hline$\pi-\gamma \leq \alpha<\pi$ & 0 & 1 & 1 & 1 & 0 \\
\hline$\alpha=\pi$ & 0 & 0 & 1 & 1 & 0 \\
\hline
\end{tabular}

point projected onto the LOS. It is function of the carrier frequency of the signal, $f$, and of the aspect angle as follows

$$
\rho_{i}=\rho_{i}(f, \alpha(t)) \simeq \frac{4 \pi f}{c}\left[x_{i} \sin \alpha(t)+z_{i} \cos \alpha(t)\right]
$$

where $\left(x_{i}, z_{i}\right)$ are the coordinates of the $i$-th scattering points onto the plane $\hat{x} \hat{z}$. The values of complex coefficients modulus for $\alpha(t) \in[\pi, 2 \pi]$ can be easily obtained thanks to the symmetry of the targets considered in this paper.

The other two mathematical models for the scatterer complex coefficients refer to two different polarizations: vertical and horizontal polarization. The mathematical expressions of the coefficients are shown in the Appendix A. The phase of the complex coefficients for this two models is evaluated with respect to a reference phase centre, which can be different from the centre of mass. Since the centre of mass is stationary with respect to the micro motions, the electromagnetic field scattered by the target is generally calculated by considering the centre of mass as the phase reference centre. For this reason, (3) for both vertical and horizontal polarization RCS model is modified considering a corrective term for the phase, as follows:

$$
\begin{aligned}
& s(n, m)= \\
& \sum_{i=1}^{N_{P}} \sigma_{i}(n, m) e^{j \rho_{i}(n, m)} e^{-j \frac{4 \pi}{c} f_{n}\left[\Delta R+d_{M P} \cos \left(\alpha_{n, m}\right)\right]} \\
& +w(n, m)
\end{aligned}
$$

where $\alpha_{n, m}=\alpha(m T+n T r), \sigma_{i}(n, m)=\sigma_{i}\left(f_{n}, \alpha_{n, m}\right)$ and $\rho_{i}(n, m)=\rho_{i}\left(f_{n}, \alpha_{n, m}\right), d_{M P}$ is the distance along the symmetric axis between the centre of mass and the phase reference centre, represented respectively by the points MC and RP in Figures $4 a, 4 b$ and $4 c$.

Figure 5 shows the normalized HRRPs (in $\mathrm{dB}$ ) obtained for a conical target varying the aspect angle over $360^{\circ}$, with $\Delta R=0$, for the three RCS models. The cone height and diameter are $1 \mathrm{~m}$ and $0.7 \mathrm{~m}$, respectively. It is worth mentioning that the HRRPs are simulated under the hypothesis that the object is stopped during the acquisition of each burst and in absence of noise, in order to analysis only the variation of HRRP of the target over the aspect angle. This means that the aspect angle is considered constant during the burst, 
such that $\alpha_{n, m}=\alpha_{m}=\alpha(m T)$. A SFWs radar with a total bandwidth of $800 \mathrm{MHz}$ between 2.6 and 3.4 $\mathrm{GHz}$ is considered, transmitting 128 sub-pulses with a PRF of $20 \mathrm{KHz}$. The range resolution guaranteed by the considered radar is $18.75 \mathrm{~cm}$. For each value of aspect angle the received signal vector is zero-padded along the stepped frequency computing the IDFT over 512 bins to obtain the HRRP. Moreover, a Hann window is used in order to emphasize the scatterers with lower coefficient modulus in the vertical and the horizontal polarization. Observing Figure $5 \mathrm{~b}$ and Figure $5 \mathrm{c}$ it is noted that the contribution of the cone tip in the scattered field is generally lower than the contribution of the scatterers on the bottom, in both polarizations. However in a small interval of values of aspect angle (see from $0^{\circ}$ to around $45^{\circ}$ ), the tip of the cone is more visible in the vertical polarization than in the horizontal. Moreover, it is worth noting that the HRRP cannot be calculated for some values of the aspect angle with both vertical and horizontal polarization models due to the approximations considered in these RCS models (see vertical spikes in the HRRP frames shown in Figure 5b and Figure 5c). Let us consider as example the approximation for the cone which occurs for incidence nearly perpendicular to the base, specifically when $\alpha \in\left[\pi-\alpha_{c a}, \pi\right]$, with $\alpha_{c a}=\alpha_{c a}(f)$ the axial crossover angle which varies on the carrier frequency (see the Appendix). Figure 6 shows how the normalized RCS of the cone for $\alpha=177^{\circ}$ and the bound $\pi-\alpha_{c a}$ for the approximation vary on the carrier frequency. It is noted that for frequencies smaller than $3 \mathrm{GHz}$ the approximation occurs, while it does not for greater frequencies. This leads to a discontinuity in the RCS of simulated wide-band echo which does not allow to obtain a correct HRRP. All the details on the RCS models for both the two polarization are well described in [20].

Figure 7 shows the normalized HRRPs over $360^{\circ}$, with $\Delta R=0$, from a cylinder whose height and diameter are $1 \mathrm{~m}$ and $0.7 \mathrm{~m}$, respectively [17]. From Figure 7a it is noted that for each value of the aspect angle three scatterers are simultaneously visible at most. Moreover, while for the vertical polarization the scattering coefficients of some scatterers are higher then the others, with horizontal polarization the scattering contributions of visible scatterers are similar between each other (see Figure $7 \mathrm{~b}$ and Figure $7 \mathrm{c}$ ).

Figure 8 shows the normalized HRRPs over $360^{\circ}$, with $\Delta R=0$, from a target composed by a cone plus a cylinder. The cone and cylinder heights are $1.4 \mathrm{~m}$ and 0.7 $\mathrm{m}$, respectively, while the diameter is $0.4 \mathrm{~m}$ [6]. Figure $8 \mathrm{~b}$ and $8 \mathrm{c}$ show that the contribution from the cone tip is generally lower than the ones from the other scatterers.
However, even in this case the tip of the cone is more visible in the vertical polarization than in the horizontal one.

Finally it is pointed out that even for the RCS model of cylinder and cone plus cylinder for both the analysed polarizations, some approximations are considered leading to errors in the HRRP evaluation for some values of the aspect angle, as described for the cone.

Although many models for predicting the RCS for several targets are present in the literature, it is worth noting that the scattering phenomenon depends on a large number of factors e.g. the target geometry, aspect angle and altitude with respect to radar antenna, and atmospheric factors, which lead to uncontrolled scintillation of the RCS. In order to take into account these fluctuation in the signal modelling, the target RCS is usually expressed as a random variable [21]. Through some experimental analysis it has been shown in [22] that the RCS of missiles shows fluctuation which can be well represented by a log-normal random variable [23] Hence, the received signal sample $s(n, m)$ is written as:

$$
\begin{aligned}
& s(n, m)= \\
& \sqrt{g(n, m)}\left(\sum_{i=1}^{N_{P}} \sigma_{i}(n, m) e^{j \rho_{i}(n, m)}\right) \times \\
& e^{-j \frac{4 \pi}{c} f_{n}\left(\Delta R+d_{M P} \cos \left[\alpha_{n, m}\right)\right]} \\
& +w(n, m)
\end{aligned}
$$

where $g(n, m)$ is a statistical sample from log-normal distribution.

\section{HRRP FRAME BASED CLASSIFICATION ALGORITHM FOR BTS}

In this section the classification algorithm presented in [17] which is able to extract reliable feature from the HRRP frame based on the micro-motions exhibited by BTs is described. Figure 9 represents a scheme block of the presented algorithm.

\section{HRRP Frame Acquisition}

The aim of the first block is to acquire a HRRP frame whose time duration is approximately as long as the period of rotational motion exhibited by the target. Therefore an accurate estimation of main rotation period exhibited by the target is needed. In the literature there are presented several method for the estimation of rotation rate $\widehat{\Omega_{r}}$ of a target [24], [25], [26], [16]. However, the rate estimation processing is out of the scope of this work. The number $\widehat{M}$ of bursts needed for computing the target classification depends on the 


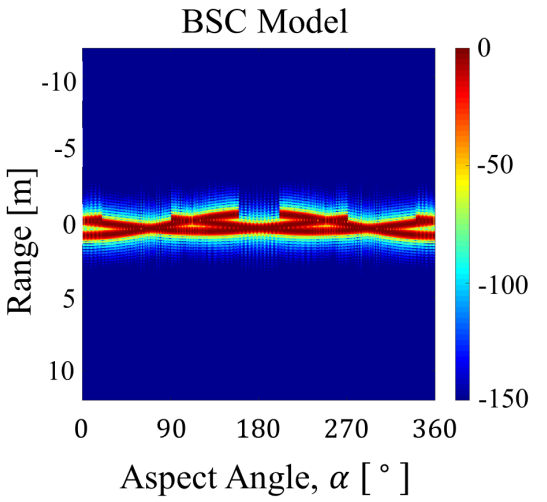

(a)

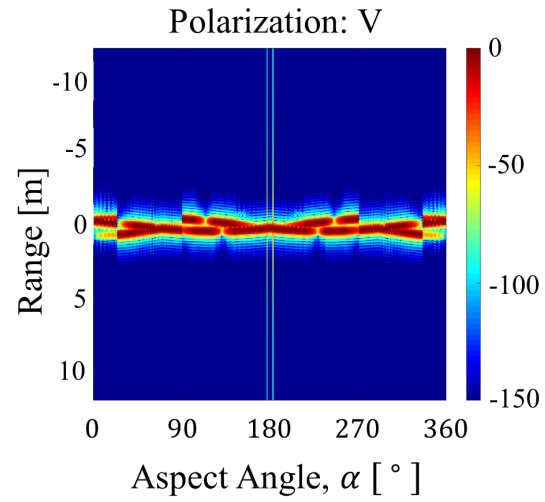

(b)

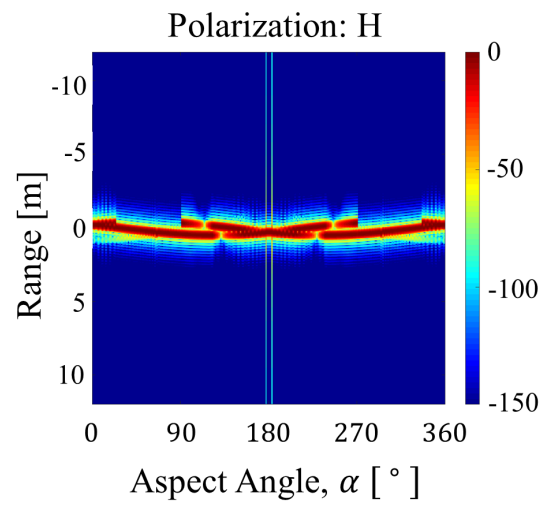

(c)

Figure 5: Normalized HRRP from the cone for $\alpha \in[0,2 \pi]$ : (a) BSC; (b) Vertical Polarization; (c) Horizontal Polarization.

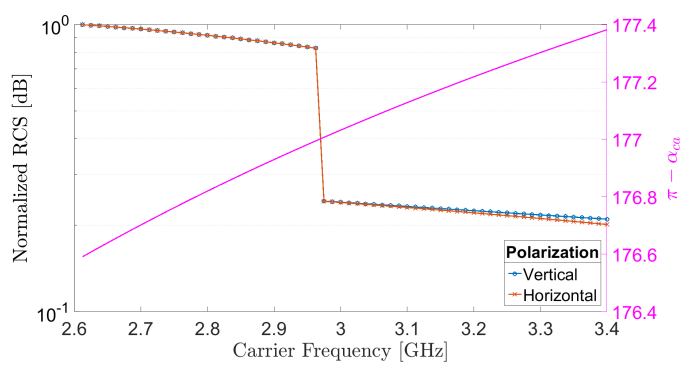

Figure 6: The bound for the cone approximation for incidence nearly perpendicular to the base; the normalized RCS of the cone when $\alpha=177^{\circ}$ on varying the frequency.

estimated rotation rate value $\widehat{\Omega_{r}}$ and the SFWs radar parameters. Specifically it follows:

$$
\widehat{M}=\left\lceil\frac{\widehat{\Omega_{r}}}{2 \pi \mathrm{BRF}}\right\rceil
$$

where BRF is the Burst Repetition Frequency, which is the number of the entire sub-pulses sequences transmitted in a second. It is worth noting that an approximation error may occur due to the fact that the number of bursts to cover an entire rotation period is not an integer.

Figure 10 represents the HRRP frame acquisition scheme, where two possible configuration are illustrated. In the first configuration (red lines in Figure 10) the estimation of the rotation rate, and consequently of the number $\widehat{M}$ of bursts making up the HRRP frame, is computed by using primary observations of the target by cooperative system. Then the SFWs radar will transmit $\widehat{M}$ bursts for generating the frame for the classification algorithm. In the second configuration (green lines in Figure 10), data acquired directly by the SFWs radar are used for the estimation of $\widehat{M}$. Then the selection data block will extract the sequence of bursts for the classification directly from the available data.

The received signals from each bursts are processed as described in Section II-A in order to obtain a HRRP frame from the target. The output of the first block is the matrix, $\chi$, whose each column contains the HRRP from a single burst.

\section{A. Signature Extraction}

The signature extraction block is composed of two sub-blocks, namely the pre-processing and the IRT block, as shown in Figure 9. The pre-processing block consists of two steps. The first is the normalization of each HRRP which makes up the frame with respect to its own maximum value:

$$
\bar{\chi}(\varepsilon, m)=\frac{\chi(\varepsilon, m)}{\max _{\varepsilon} \chi(\varepsilon, m)}
$$

The second step consists of resizing the normalized frame $\bar{\chi}$ around the range of centre of mass, $R_{M C}$, such that the interval of considered ranges is greater than the maximum dimension of the targets of interest. Following, the target signature $\boldsymbol{I}$ is obtained normalizing by $\hat{M}$ the IRT of the output of the pre-processing block, $\tilde{\chi}$, as follows:

$$
\boldsymbol{I}=\frac{\operatorname{IRT}\{\tilde{\boldsymbol{\chi}}\}}{\hat{M}}
$$

The RT computes the projection of a 2-D function onto a specific direction [10]. Considering a function $f$ and a 


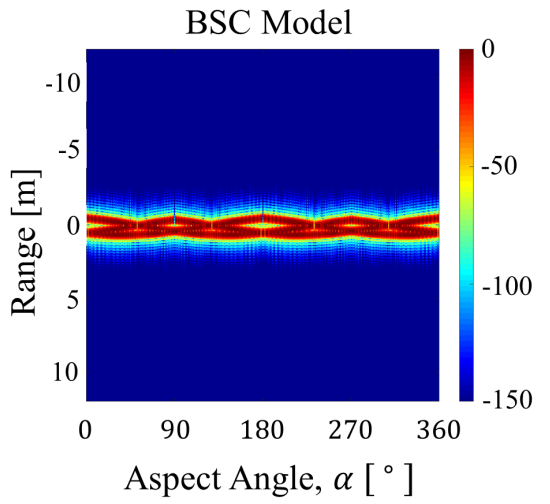

(a)

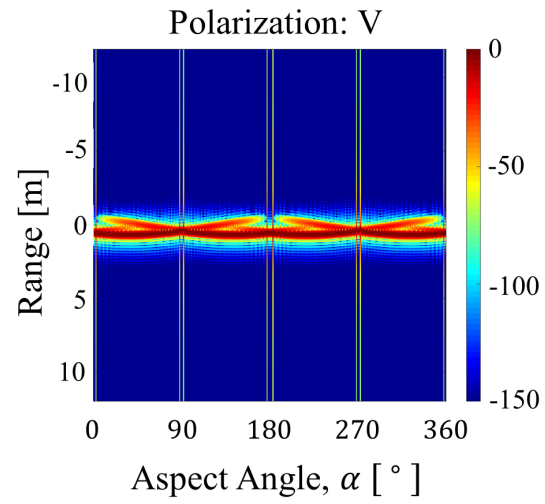

(b)

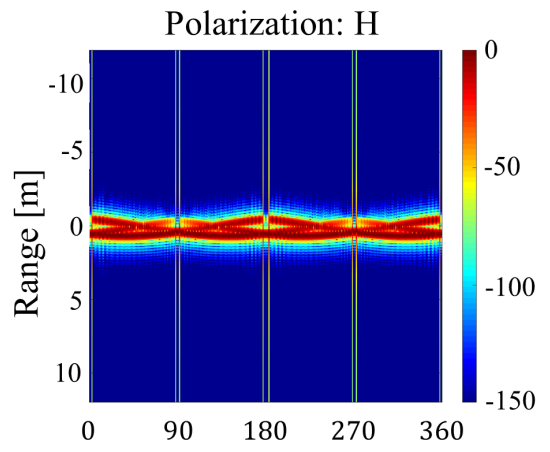

Aspect Angle, $\alpha\left[^{\circ}\right]$

(c)

Figure 7: Normalized HRRP from the cylinder for $\alpha \in[0,2 \pi]$ : (a) BSC; (b) Vertical Polarization; (c) Horizontal Polarization.

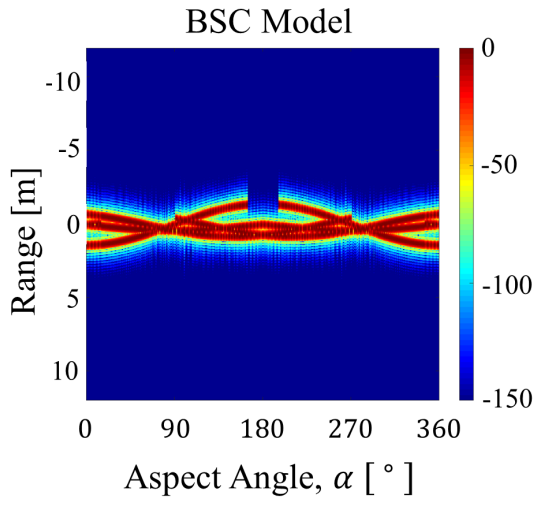

(a)

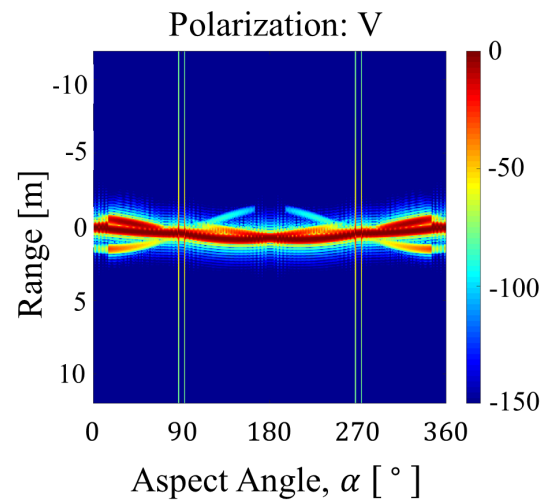

(b)

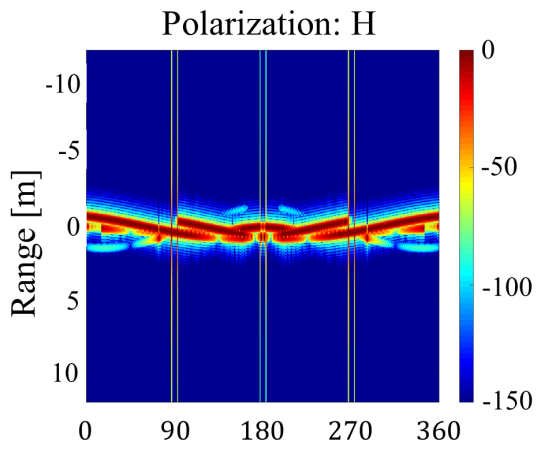

Aspect Angle, $\alpha\left[^{\circ}\right]$

(c)

Figure 8: Normalized HRRP from the cone plus cylinder for $\alpha \in[0,2 \pi]$ : (a) BSC; (b) Vertical Polarization; (c) Horizontal Polarization.

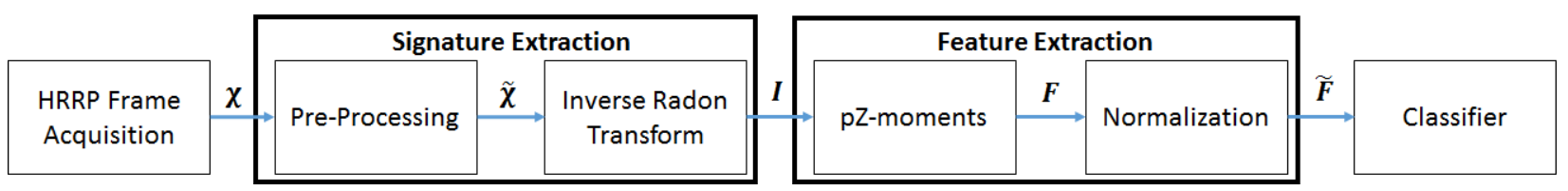

Figure 9: Algorithm block scheme.

line $L$ defined in $\mathbb{R}^{2}$, the $\mathrm{RT}$ of $f$ is $[10]$ :

$$
\mathcal{R}_{f}=\int_{L} f(x, y) d l
$$

where $x, y$ are coordinates of points on the plane, and, $d l$ is the increment of length along $L$. In order to better define the integral in (18), let us consider the definition of an arbitrary line in the normal form with respect to the coordinate system $(x, y)$, given by

$$
p=x \cos (\phi)+y \sin (\phi)
$$

with $\phi$ being the inclination angle with respect to the $x$-axis (see Figure 11). It follows that $\mathcal{R}_{f}$ depends on $p$ and $\phi$. Considering the coordinate system $(p, s)$ obtained 


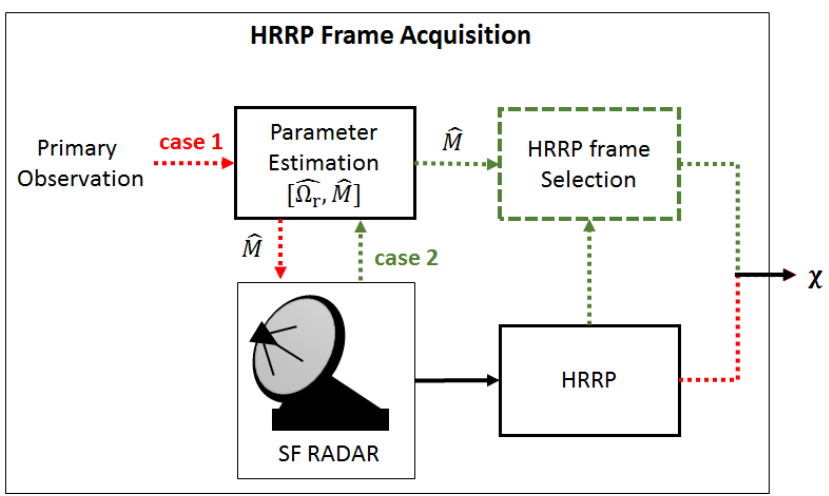

Figure 10: HRRP frame generation block scheme.

rotating the system $(x, y)$ by the angle $\phi$ such that

$$
\begin{aligned}
& x=p \cos (\phi)+s \sin (\phi) \\
& y=p \sin (\phi)+s \cos (\phi)
\end{aligned}
$$

the RT of $f$ can be written as:

$$
\begin{aligned}
& \mathcal{R}_{f}=\mathcal{R}_{f}(p, \phi)= \\
& \int_{-\infty}^{\infty} f(p \cos (\phi)+s \sin (\phi), p \sin (\phi)+s \cos (\phi)) d s
\end{aligned}
$$

where the limits may be finite if the function $f$ is zero outside its domain $\mathcal{D}$.

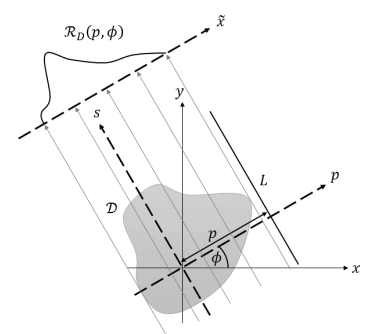

Figure 11: Example of function $f(x, y)$ domain $\mathcal{D}$ and a generic line $L$ (black line) in the original (continuous lines) e rotated (dashed lines) reference coordinate system.

Let us consider the easier case in which the 2-D function is given by a delta function located at the point $\left(x_{0}, y_{0}\right)$ as follows

$$
f(x, y)=\delta\left(x-x_{0}\right) \delta\left(y-y_{0}\right)
$$

Then its RT onto the line in (19) is

$$
\begin{aligned}
\mathcal{R}_{f}(p, \phi)= & \int_{L} \delta\left(x-x_{0}\right) \delta\left(y-y_{0}\right) d s= \\
& \int_{-\infty}^{\infty} \delta\left(p-p_{0}\right) \delta\left(s-s_{0}\right) d s= \\
& \delta\left(p-x_{0} \cos (\phi)-y_{0} \sin (\phi)\right)
\end{aligned}
$$

where

$$
\begin{aligned}
& p_{0}=x_{0} \cos (\phi)+y_{0} \sin (\phi) \\
& s_{0}=x_{0} \sin (\phi)+y_{0} \cos (\phi)
\end{aligned}
$$

It is noted that the RT of a delta function in $\mathbb{R}^{2}$ generates a sinusoidal pattern in the 2-D domain $(p, \phi)$ as follows:

$$
p=A \sin \left(\phi+\phi_{0}\right)
$$

with

$$
\begin{aligned}
A & =\sqrt{x_{0}^{2}+y_{0}^{2}} \\
\phi_{0} & =\tan ^{-1}\left\{\frac{x_{0}}{y_{0}}\right\}
\end{aligned}
$$

For this reason the data obtained by the RT is known as sinogram [27]. By contrast the IRT allows to reconstruct a 2-D function from its projections converting any sinusoidal pattern into a point.

The space distribution function of principal target scatterers is a 2-D function defined on the plane $\hat{x} \hat{z}$ given by the superimposition of delta functions as follows:

$$
\mathcal{F}=\sum_{i=1}^{N_{p}} \delta\left(x-x_{i}\right) \delta\left(z-z_{i}\right)
$$

where $\left(x_{i}, z_{i}\right)$ are the coordinates of the $i$-th scattering points onto the plane $\hat{x} \hat{z}$. In the hypothesis that the principal motion of the target is compensated, the range of each scatters $R_{i}$ in the HRRP frame depends on the aspect angle as follows:

$$
\begin{aligned}
& R_{i}(t) \\
& =\Delta R-x_{i} \sin \alpha(t)-z_{i} \cos \alpha(t) \\
& =\Delta R-\sqrt{x_{i}^{2}+z_{i}^{2}} \sin \left[\alpha(t)+\tan ^{-1}\left\{\frac{x_{i}}{y_{i}}\right\}\right]
\end{aligned}
$$

Figure 12 shows the range maps and their IRT for the three scatterers of a cone considering an entire rotation period, $T_{r}$, for different couple of values of $(\beta, \theta)$. The micro-motions exhibited by target leads to periodic tracks in the range-slow time domain. Specifically each scattering point generates a sinusoidal path centred at $\Delta R$ in the HRRP frame when $\alpha(t)$ varies into $[0, \pi]$. Then, applying the IRT, all the energy recovered from the path of a single scatterer is concentrated into a point obtaining an image which represents the profile of the object with the exact relative distances between scatterers onto plane $\hat{x} \hat{z}$ (ISAR image of the object). However, from (7) it is clear that $\alpha(t)$ generally varies sinusoidally into $[|\theta-\beta|,|\theta+\beta|]$. So each scatterer generally moves on a different periodic path. In this specific case, by applying the IRT, the energy from each path is dispersed into the final 2-D image, such that each of them generates a close 
line, e.g. circumference or ellipse. For example, Figure $12 \mathrm{e}$ shows the IRT of the range map from a tumbling cone with $(\beta, \theta)=\left(60^{\circ}, 90^{\circ}\right)$, where the contribution from the cone tip is concentrated in a point and the points on the base generate an ellipse, while Figure $12 \mathrm{f}$ shows the IRT of the range map from a precessing cone with $(\beta, \theta)=\left(60^{\circ}, 10^{\circ}\right)$, in which each scatterer leads to a different circumference. Therefore, the IRT of HRRP frame can represent the target signature since the close lines are strictly related to the coordinates of scattering points onto the plane $\hat{x} \hat{z}$.

\section{B. Feature Extraction}

The pZ moments are geometrical moments with several properties, among which is that their modulus is rotational invariant. Therefore, in this work the $\mathrm{pZ}$ moments of the target signature $\boldsymbol{I}$ are computed in order to extract the feature vector.

Introduced in [28], the $\mathrm{pZ}$ moments of order $o$ and repetition $l$ of a 2-D image $\mathcal{I}(x, y)$ are calculated by projecting the image on a basis of 2-D polynomials which are defined on the unit circle as follows:

$$
\begin{aligned}
& \zeta_{o, l}= \\
& \frac{o+1}{\pi} \int_{0}^{2 \pi} \int_{0}^{1} W_{o, l}^{*}(\rho, \theta) \mathcal{I}(\rho \cos \theta, \rho \sin \theta) \rho \mathrm{d} \rho \mathrm{d} \theta
\end{aligned}
$$

where

$$
\begin{aligned}
& W_{o, l}(\rho, \theta)= \\
& \sum_{i=0}^{o-|l|} \frac{\rho^{r-i}(-1)^{i}(2 o+1-i) !}{i !(o+|l|+1-i) !(o-|l|-i) !} e^{j l \theta} \\
& \text { with } \quad \rho \leq 1 .
\end{aligned}
$$

The presented algorithm computes $(O+1)^{2}$ pZ moments, where $O$ is the maximum order by projecting $\hat{\boldsymbol{I}}$ on the $\mathrm{pZ}$ polynomials, and obtaining a feature vector whose $z$-th element is:

$$
F_{z}=\left|\zeta_{o, l}\right|
$$

where $o=l=0, \cdots, O-1$ and $z=0, \cdots,(O+1)^{2}-1$. Since the $\mathrm{pZ}$ moments are defined on the unit circle, the signature $\tilde{\boldsymbol{I}}$ is inscribed in the unit circle [18]. Finally, the feature vector given by

$$
\boldsymbol{F}=\left[F_{0} F_{1} \cdots F_{\mathcal{Z}-1}\right] .
$$

with $\mathcal{Z}=(O+1)^{2}$, is statistically normalised in order to avoid that polarized vector may affect the classification process. Hence, the final vector input to the classifier is:

$$
\tilde{\boldsymbol{F}}=\frac{\boldsymbol{F}-\eta_{\boldsymbol{F}}}{\varsigma_{\boldsymbol{F}}}
$$

where $\eta_{\boldsymbol{F}}$ and $\varsigma_{\boldsymbol{F}}$ are the statistical mean and the standard deviation of the vector $\boldsymbol{F}$, respectively.

\section{Classifier}

Classifiers are mathematical techniques designed to compare the extracted features within a database, which contain the information of all the targets of interest. In this paper, the classification performances of the extracted feature vectors are evaluated using the $k$-Nearest Neighbour $(k \mathrm{NN})$ classifier. The $\mathrm{kNN}$ classifier is chosen as it is based on the evaluation of the Euclidean distances between the vector under test and the vectors composing the training set of each class in order to estimate the target class. Hence, the classification performance evaluated with $k \mathrm{NN}$ classifier are not polarized by the properties of the classifier, and it depends only on the characteristic of features to occupy multidimensional spaces for each class sufficiently separated. However, in general other classifiers with similar characteristics could be also selected. The selection of the best classifier is outside the scope of this paper.

\section{Micro-Motion Velocity EFFECT ON TARGET SIGNATURE}

In presence of a target which moves with a radial velocity $v_{r}$ along the LOS, the target range varies of about $2 N v_{r} T$ within the burst acquisition. The bulk motion velocity of the target introduces a phase term, which is the major cause of distortion for the HRRP, leading to a reduced peak response and the occurrence of side-lobes. In the same way, the variation of aspect angle during the acquisition of each burst, which depends on the velocity of scatterers motion with respect to the centre of mass, represents an additional distortion factor. Let us assume that the target is tracked and the main Doppler shift due to the bulk motion is compensated perfectly, such that $\Delta R=R_{M C}(t)-R_{0}(t)=0$. From these assumption follows that the HRRP frame shows how the distance between the radar and each principal scattering point of the target changes with time due to the micro-motions. The peak value of the range profile for each scatterer of the target locates at:

$$
\frac{4 \pi}{c} \Delta f \hat{R}_{i}=-\frac{4 \pi}{c} \Delta f R_{i}-\frac{4 \pi}{c} \frac{f_{0} v_{i} T}{\Delta f}
$$

where $R_{i}$ is the projection of the distance between the $i$-th scatterer and the centre of mass along the LOS, and $v_{i}$ is the velocity of the $i$-th scatterer due to the micromotion. It is worth noting that the micro-motion of a target leads to a multi-targets (scatterers) scenario, in which each of them has a different velocity profile, given by

$$
v_{i}=v_{i}(t)=\left(x_{i} \sin \alpha(t)-z_{i} \cos \alpha(t)\right) \frac{\mathrm{d} \alpha(t)}{\mathrm{d} t}
$$




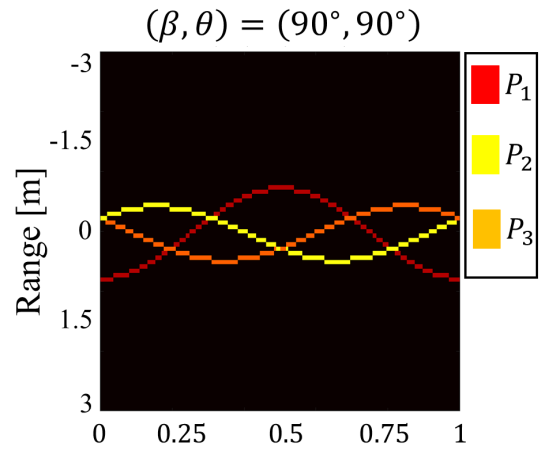

Normalised Rotation Period [s]

(a)

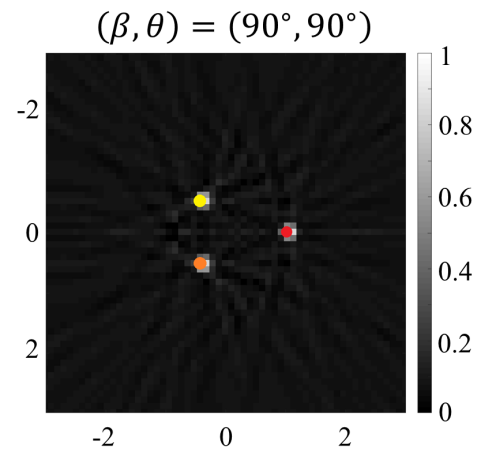

(d)

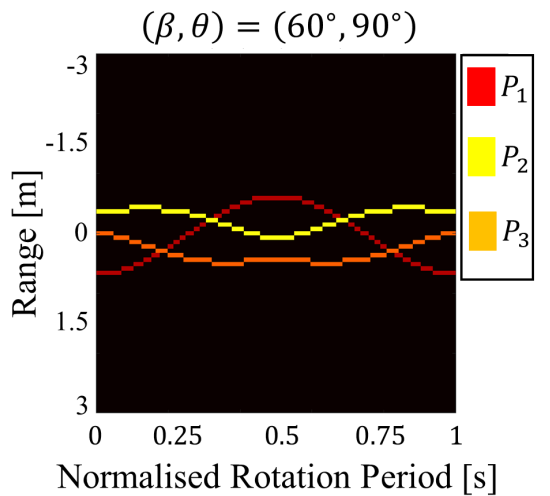

(b)

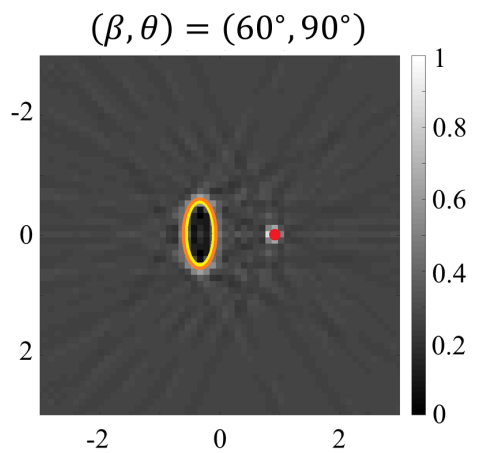

(e)

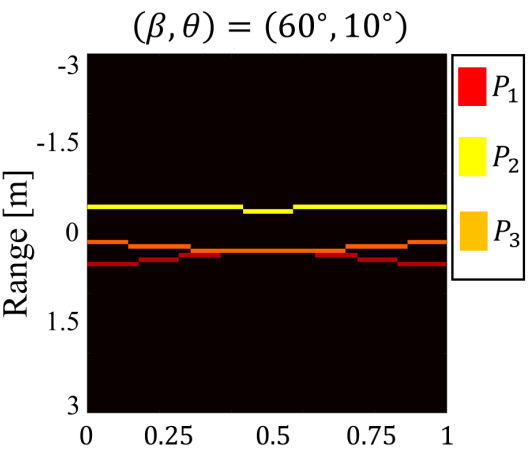

Normalised Rotation Period [s]

(c)

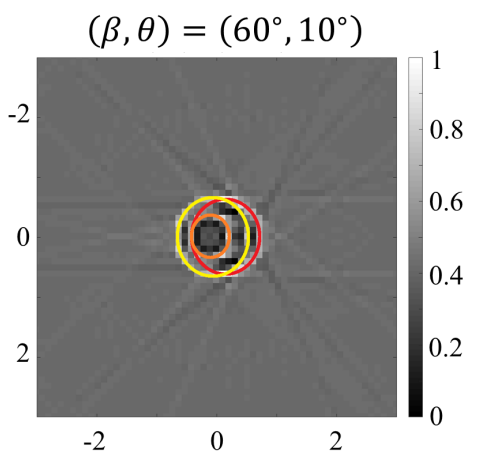

(f)

Figure 12: Range map and its IRT for the three points of cone considering a whole rotation period, $T_{r}$, for different couple of values of $(\beta, \theta)$ : (a) range map for a complete rotation of cone with $(\beta, \theta)=\left(90^{\circ}, 90^{\circ}\right)$, (b) range map tumbling cone with $(\beta, \theta)=\left(60^{\circ}, 90^{\circ}\right)$, (c) range map for precessing cone with $(\beta, \theta)=\left(60^{\circ}, 10^{\circ}\right)$; (d),(e) and (f) are the IRT of the range maps (a),(b) and (c), respectively.

with

$$
\begin{aligned}
& \frac{\frac{\mathrm{d} \alpha(t)}{\mathrm{d} t}=}{\Omega_{r} \sin (\beta) \sin (\theta) \cos \left(\Omega_{r} t+\phi\right)} \\
& \sqrt{1-\left(\sin (\beta) \sin (\theta) \cos \left(\Omega_{r} t+\phi\right)+\cos (\beta) \cos (\theta)\right)^{2}}
\end{aligned}
$$

Hence, the displacement from the effective range for each scatterer is different according to its position on target surface, the target motion and the radar position. Figure 13 shows an example of how HRRP frame from the three considered shapes varies considering the stop-and-go hypothesis (dash line) and the continuous motion during the burst acquisition (continuous line). In the example shown, $(\beta, \theta)=\left(90^{\circ}, 90^{\circ}\right)$ and $\Omega_{r}=6 \pi$. Moreover, the occlusion and the polarization scattering properties of the scatterers are not taken into consideration in order to only demonstrate the micro-motion effect on the HRRPs. It is worth noting that a rotational motion leads to a circular shift of the tracks of each scatterer in the frame. This shift leads to a rotation of the 2D image recovered by using the IRT. Additionally, the maximum range of each scatterer is greater with respect to the real value, such that the object appears greater in the target signature. However, since the velocity of each scatterer depends on the geometry of the target and their distances from target centre of mass, the signature shape (hence, the target shape) may appear distorted, e.g. the conical shapes appear with a greater hight and base ratio. Finally, since a rotation leads to an harmonic radial velocity, the velocity is even not constant during the burst. Specifically, acceleration affects the HRRP reducing the SNR on the 2D target signature. 


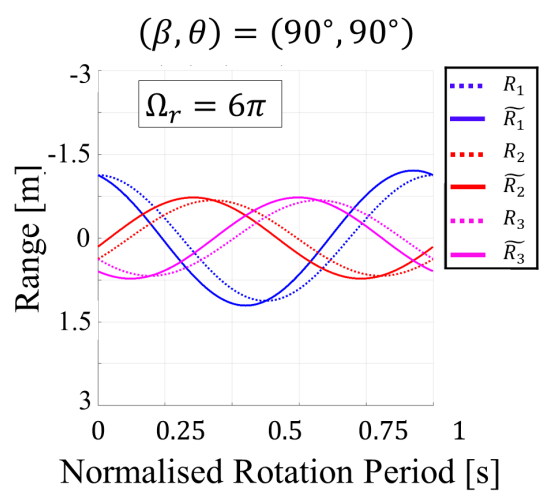

(a) Cone

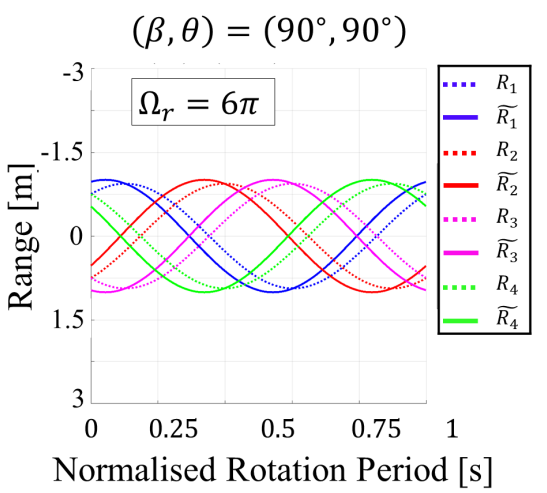

(b) Cylinder

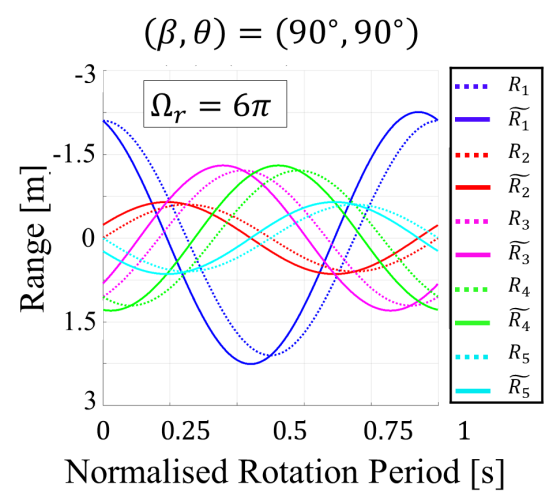

(c) Cone plus Cylinder

Figure 13: Example of HRRP frame from the three considered shapes considering the stop-and-go hypothesis (dashed line) and continuous motion during the burst acquisition (continuous line), for $(\beta, \theta)=\left(90^{\circ}, 90^{\circ}\right)$ and $\Omega_{r}=6 \pi$.

The pZ moments based features guarantee robustness against rotational and scale effects on the target signature. However, in order to reduce the deforming effect due to the micro-motion and to improve the classification capabilities, the radar SFW may be adaptive to the estimated rotation rate.

\section{Performance Analysis}

In this section the performance of the proposed classification algorithm is evaluated with simulated data. The selected parameters (targets' sizes, carrier frequency, bandwidth, etc.) have been selected in agreement to what is available in the literature [4], [6], [17], [29], [30] and on the experience of the author's in the field being involved in research projects on the topic in the past.

The algorithm is tested considering three possible shapes for the BTs which are the cone, the cylinder and the cone plus cylinder. The cone and the cylinder have the same height and radius which are $1 \mathrm{~m}$ and $0.375 \mathrm{~m}$, respectively [17]. The third shape is obtained by joining a cone whose height and radius are $1.4 \mathrm{~m}$ and $0.2 \mathrm{~m}$, respectively, and a cylinder with a height of $0.7 \mathrm{~m}$ and radius $0.2 \mathrm{~m}$ [6]. Table IV synthesizes the dimensions of the targets of interest.

Table IV: Target Dimensions.

\begin{tabular}{|l|c|c|c|}
\hline & $h_{1}[\mathrm{~m}]$ & $h_{2}[\mathrm{~m}]$ & $r[\mathrm{~m}]$ \\
\hline Cone & 0.750 & 0.250 & 0.375 \\
\hline Cylinder & 0.500 & 0.500 & 0.375 \\
\hline Cone plus Cylinder & 1.400 & 0.700 & 0.200 \\
\hline
\end{tabular}

Six classes are considered, each of them corresponding to a particular shape and motion:

1) precessing cone;

2) tumbling cone;

3) precessing cylinder;

4) tumbling cylinder;

5) precessing cone plus cylinder;

6) tumbling cone plus cylinder;

Generally the precession angle of warheads with a conical shape is relatively small compared to the half cone angle [4] and its value is generally within $\left[4^{\circ}, 12^{\circ}\right]$ [29]. In this work the precessing classes for each shape are obtained by fixing the precession angle $\theta$ equal to $10^{\circ}$, while for the tumbling classes $\theta=90^{\circ}$.

Both the training and testing sets are simulated considering a SFWs radar transmitting bursts composed by 128 sub-pulses with a total bandwidth of $800 \mathrm{MHz}$ and a PRF of $20 \mathrm{kHz}$. All the SFWs radar parameters are synthesized in Table V.

Table V: SFWs radar system parameters.

\begin{tabular}{|lc|c|}
\hline Carrier frequency & {$[\mathrm{GHz}]$} & 2.600 \\
\hline Total bandwidth & {$[\mathrm{MHz}]$} & 800 \\
\hline Number of sub-pulses & $N$ & 128 \\
\hline Waveform bandwidth & {$[\mathrm{MHz}]$} & 6.25 \\
\hline Pulse Repetition Interval & {$[\mathrm{kHz}]$} & 20 \\
\hline Burst Repetition Interval & {$[\mathrm{Hz}]$} & 156.25 \\
\hline
\end{tabular}

The training set for each class is realized for different values of the radar position angle $\beta_{u}$ as follows

$$
\beta_{u}=u 5^{\circ} \quad \text { with } u=1,2, \cdots, 18 .
$$

Each sample of training set is obtained considering the target stopped during the acquisition of a single burst 
and in absence of noise. Specifically, for each $\beta_{u}$ a 360 long HRRP frame is simulated such that the target has completed a rotation of $1^{\circ}$ between two sequential bursts with respect to its motion. Finally the initial phase of rotation is set equal to zero.

The testing set is realized considering noisy observations and continuously moving targets, even during a single burst. In particular, since the warhead spinning and decoy tumbling frequencies are typically smaller than $3 \mathrm{~Hz}$ [30], the dataset for testing each class is realized on varying the rotation rate within $[0.25,3] \mathrm{Hz}$. Specifically the angular rotation velocities considered are

$$
\Omega_{r_{v}}=2 \pi\left[\frac{1}{4}+\frac{v}{8}\right] \frac{\mathrm{rad}}{\mathrm{s}} \quad \text { with } v=0, \cdots, 22
$$

From (15) it is pointed out that the HRRP frame length decreases as the rotation rate increases. Figure 14 shows how the number of bursts of the frame varies with the rotation rate for the SFWs radar described above. The

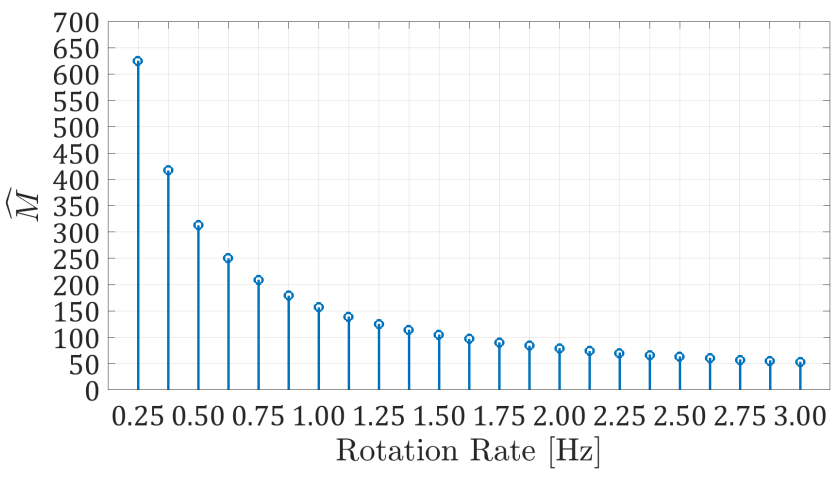

Figure 14: Number of bursts to obtain the HRRP frame on varying the angular rotation velocity and for the SFWs radar described in Table $\mathrm{V}$

testing set for each class and for a fixed noise power and rotation rate is composed by 180 samples. Each set is obtained by simulating 20 acquisitions for each value of $\beta_{\epsilon}=\epsilon 10^{\circ}$ with $\epsilon=1,2, \cdots, 9$, which are different for the noise observation and for the initial phase of the micro-motions. The initial phase is drawn randomly from a uniform distribution $[0,2 \pi]$.

The performance of the proposed algorithm is evaluated in terms of: Probability of correct Motion identification $\left(P_{M}\right)$, which represents the capability to distinguish between precessing and tumbling targets; Probability of correct Shape identification $\left(P_{S}\right)$, which represents the capability to distinguish between the different shapes of targets; Probability of correct Classification $\left(P_{C}\right)$, the capability to identify the motion and the actual shape of the target.
The analysis is conducted on varying the Signal to Noise power Ratio (SNR), referring to the noise power at the output of the stretch processing, and considering the RCS oscillation according to the lognormal distribution with unit mean and variance equal to 0.4 [16]. Figure 15 shows an example of the effect of RCS logarithm fluctuation on a sequence of HRRP from a cone for $\alpha \in[0,2 \pi]$, simulated using the BSC model without AWGN. It is worth noting that the fluctuation of the RCS during the acquisition of the entire burst leads to an increment of the level of the noise. The mean of the

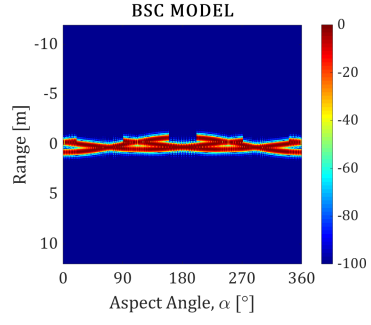

(a)

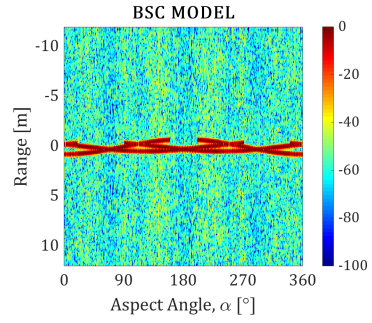

(b)
Figure 15: Example of the effect of RCS logarithm fluctuation on a sequence of HRRPs from a cone for $\alpha \in[0,2 \pi]$, simulated using the BSC model without AWGN: (a) no fluctuation; (b) RCS logarithm fluctuation.

three probabilities for each couple of values of SNR and rotation rate is evaluated with a Monte Carlo approach over $10^{4}$ different runs in which 100 samples for each class are randomly taken from the testing dataset and classified. The $k$ value of the $k$-NN classifier is chosen equal to 1 .

Figure 16 shows the performance obtained on varying the SNR and the angular rotation rate, considering the BSC model. In order to reduce the distortion in the HRRP due to the variation of the aspect angle within the burst interval a Hann window is used. It is observed that the performance in terms of the three probabilities increases as the SNR increases and decreases as the rotation velocity increases. The main reason is that the IRT integrates incoherently the HRRPs of the frame, increasing the SNR of the final image. The incoherent processing gain depends on the frame dimension: longer is the HRRP frame, higher is the processing gain. However, Figure 16a shows that $P_{S} \geq 0.99$ for SNR greater than $-5 \mathrm{~dB}$ for all the considered rotation rates. $P_{C}$ and $P_{M}$ are very similar for SNR greater than -5 $\mathrm{dB}$ since $P_{S}$ is close to 1 . Specifically, for these SNR values $P_{C}$ and $P_{M}$ varies within $[0.93,0.95]$ for all the rotation rates. It is worth noting that the performance 


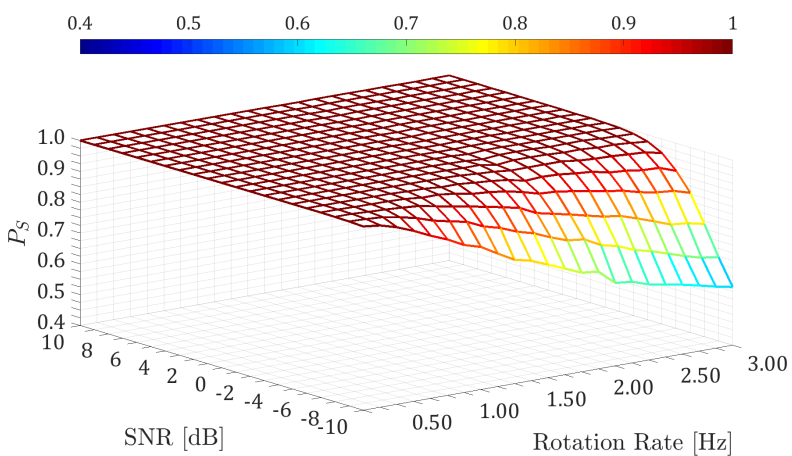

(a)

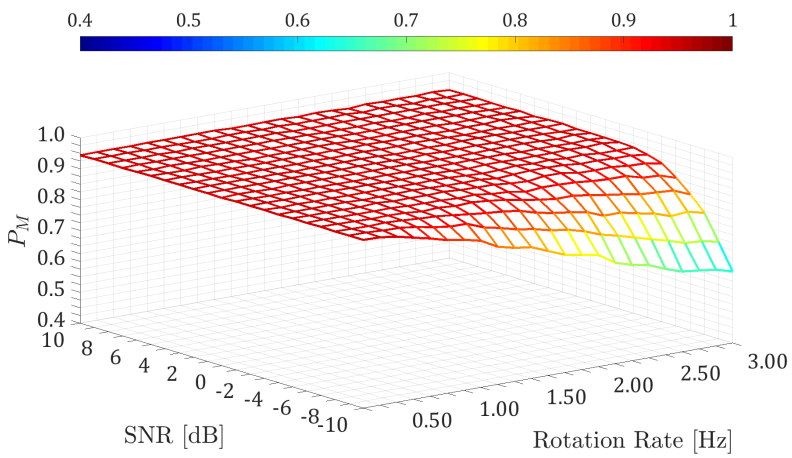

(b)

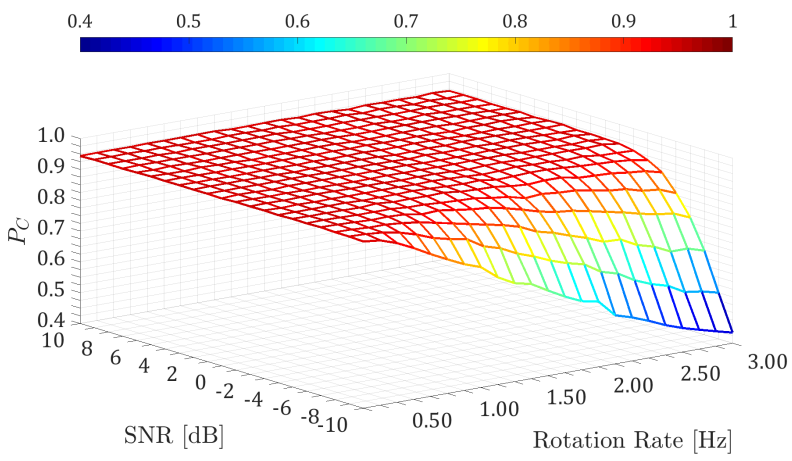

(c)

Figure 16: Performance in terms of $P_{S}$ (a), $P_{M}$ (b) and $P_{C}$ (c) by using the BSC model for the RCS.

in terms of motion recognition and correct classification are affected by the fact that the aspect angle varies in the same way when the values of the angles $\beta$ and $\theta$ are switched. In this analysis there is a case in which precession and tumbling lead to the same variation of aspect angle: since the training set for each class is composed by 18 feature vectors, the ambiguity in the motion classification is around $1 / 18 \approx 5.5 \%$. Hence, the maximum value reachable for $P_{M}$ is close to 0.95 .

Figure 17 shows the performance obtained on varying SNR and angular rotation rate, considering the vertical polarization RCS model. Similar to the previous case, Hann window is used to reduce the distortions due to the variation of the aspect angle within the burst interval and to increase the capability to observe scatterers with lower coefficients. From the results, it is observed that

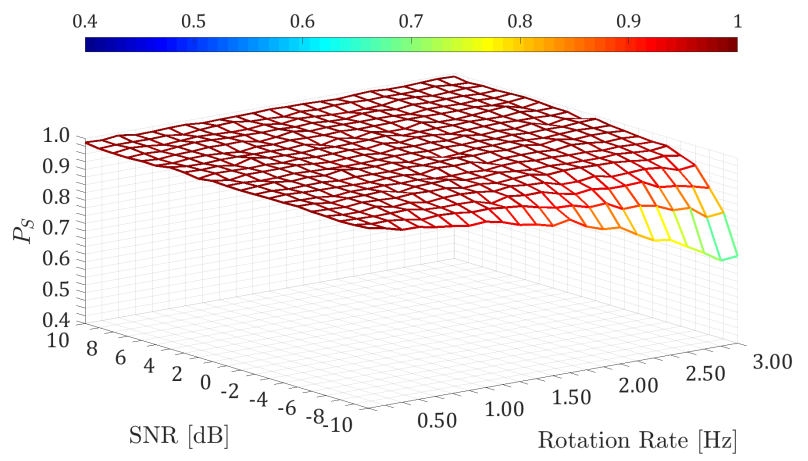

(a)

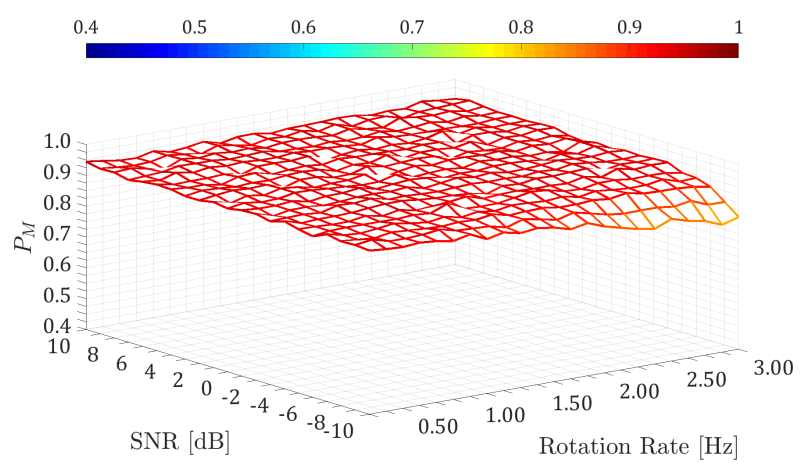

(b)

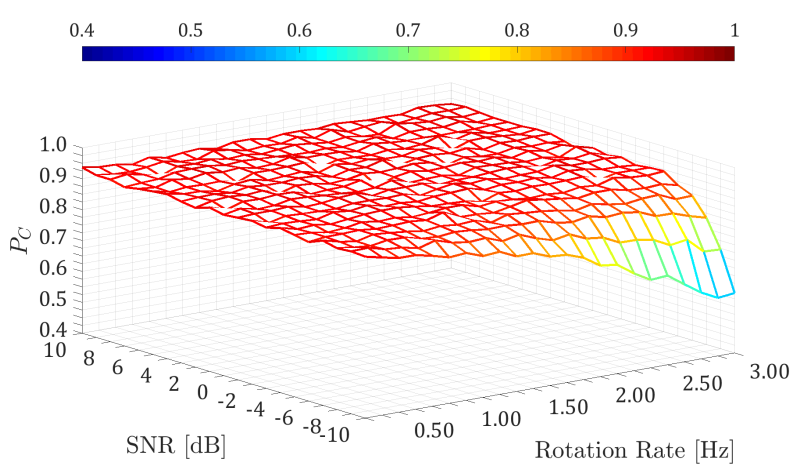

(c)

Figure 17: Performance in terms of $P_{S}$ (a), $P_{M}$ (b) and $P_{C}$ (c) by using the RCS model for vertical polarization.

the performance obtained with the vertical polarization model confirms the trend observed in Figure 16 for the BSC model. Figure 17a shows that $P_{S}>0.97$ for all the considered rotation rates when the SNR is greater than $-5 \mathrm{~dB}$, reaching a maximum value of about 0.99. Figure $17 \mathrm{~b}$ and Figure $17 \mathrm{c}$ show that $P_{M}$ varies 
within $[0.92,0.95]$ and $P_{C}$ varies within $[0.91,0.94]$ for all the considered rotation rates when the SNR is greater than $-5 \mathrm{~dB}$. Moreover, it is observed that the performance for lower values of SNR and higher rotation rates obtained with the RCS model for vertical polarization are better than the ones for the BSC model. The scattering coefficients for the RCS model described in [20] takes into consideration the target shape not only in terms of distances between the scatterers, but also of its characteristics about shape flatness and sharpness. This information may have particular importance into processing of data, especially with very low SNR values.

Figure 18 shows the performance obtained on varying SNR and angular rotation rate, considering the RCS model for the horizontal polarization. Even in this case a Hann window is used to emphasize the scatterers with lower coefficients. From Figure 18a it is observed that the capability to discriminate between the different target shapes decreases lightly by using horizontal polarization rather than the vertical polarization. The main reason is due the scattering properties of points in proximity of the sharpest parts of the object. In particular, the tips of the cone and the cone plus cylinder are more visible using the vertical polarization rather than the horizontal, in agreement with the mathematical model in [20]. However, $P_{S}$ varies within $[0.94,0.96]$ when the SNR is greater than $-2 \mathrm{~dB}$, for all the considered values of the rotation rate. The performance in terms of $P_{M}$ are similar for both the polarization models (observing Figure $17 \mathrm{~b}$ and Figure $18 \mathrm{~b}$ ), varying within [0.92, 0.95] for all the considered rotation rates when the SNR is greater than $-5 \mathrm{~dB}$. The loss in the performance in terms of $P_{S}$ using horizontal polarization leads to a loss in $P_{C}$, which varied within $[0.875,0.905]$ when the SNR is greater than $-3 \mathrm{~dB}$. Finally, it is pointed out that using the RCS model for horizontal polarization results to better performance than the ones using the BSC model for lower values of SNR and higher rotation rates.

The rotation rates of precession and tumbling are generally different. In fact while the warhead spinning and the decoy tumbling frequency may be similar, the precession frequency is typically an order of magnitude smaller with respect to the spinning [4]. Therefore, the system capability in terms of motion recognition may be improved considering also the estimated rotation velocity. For this reason the capability to recognize the target shape is considered the most relevant in this analysis. In fact the identification of the shape may be discriminant between warheads and decoys allowing also to understand which kind of warheads the target can be (cone plus cylinder can represent a warheads with an additional booster for manoeuvring).

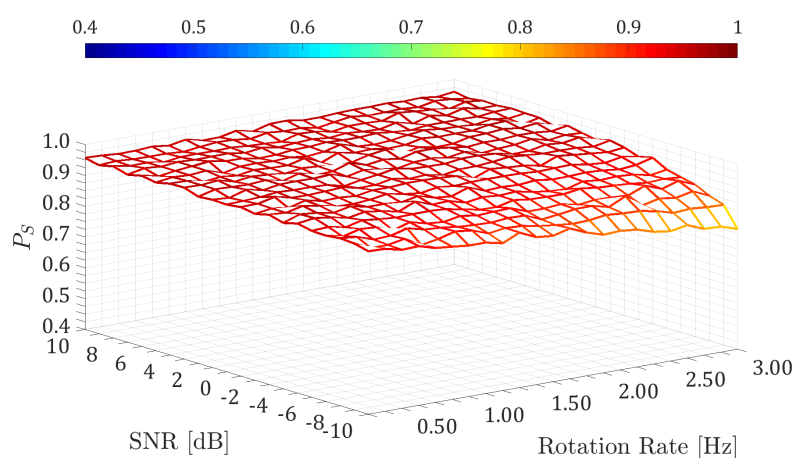

(a)

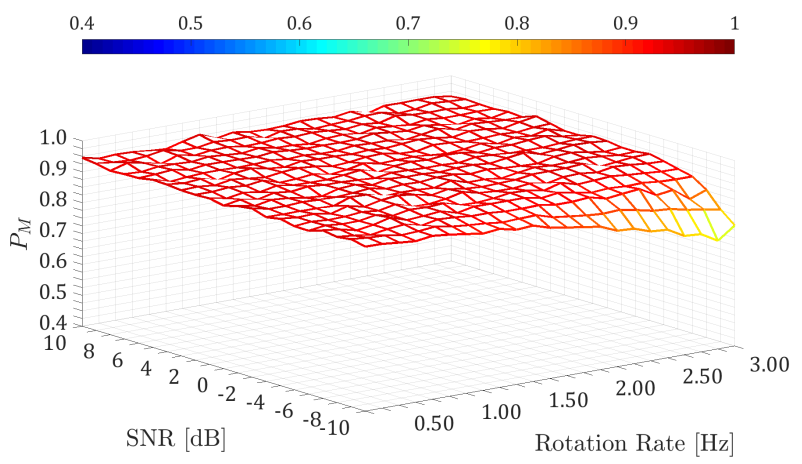

(b)

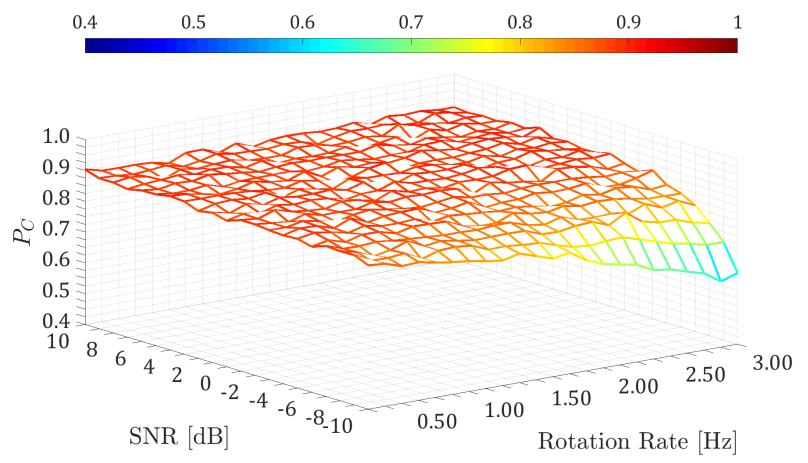

(c)

Figure 18: Performance in terms of $P_{S}$ (a), $P_{M}$ (b) and $P_{C}$ (c) by using the RCS model for horizontal polarization.

Finally it is important to point out that the classification algorithm is independent on initial phase of micro-motion and robust with respect to the receiver noise, the RCS scintillation and the approximation error on the HRRP frame dimension.

\section{A. Random Burst Repetition Frequency}

The authors in [31] present an analysis on the capability of extracting sinusoidally modulated signal parameters by using the IRT based on time-frequency 
distribution with partial data available. In fact, using the IRT as a back-projection method, it is necessary to know the angular step computed by the target between two sequentially acquisitions. For this reason, once the rotation rate of the target is estimated and, knowing the transmission time instants of each burst, it is possible to apply the algorithm proposed in the Section III, by using a subset of HRRPs which compose the frame covering the rotation period. It is worth noting that the use of a subset of HRRPs does not require any modification in the proposed classification framework, but will only effect the set of angles in which the IRT is applied.

Figure 19a shows a HRRP frame of 180 bursts considering a whole rotation period of a cone obtained for $(\beta, \theta)=\left(90^{\circ}, 90^{\circ}\right)$ considering the BSC model. Moreover, the SNR of the received signal is set equal to $10 \mathrm{~dB}$. The SFW radar parameters used for simulating the acquisition are shown above, in Table V. Figure 19b emphasizes 36 bursts randomly taken from the original frame in Figure 19a, while Figure 19c and Figure 19d shows the target signatures obtained by applying the IRT on the frame in Figure 19a and Figure 19b, respectively. It is worth noting that the signature obtained from the 36

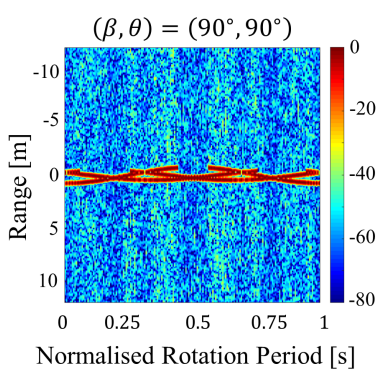

(a)

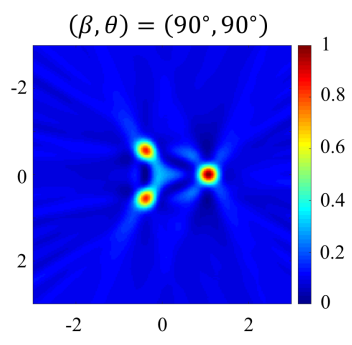

(c)

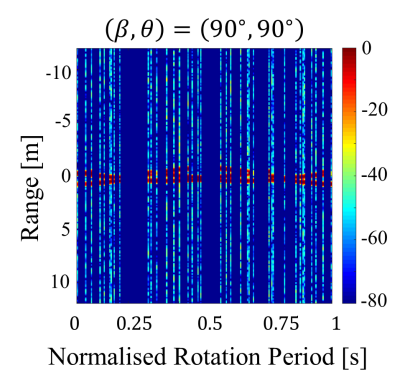

(b)

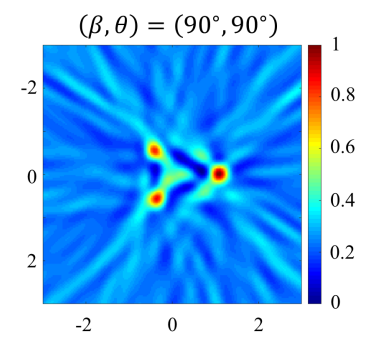

(d)
Figure 19: HRRP frame (a) and its IRT (b) of the three points of cone for BSC model obtained from a whole rotation period and $(\beta, \theta)=\left(90^{\circ}, 90^{\circ}\right)$; HRRP frame (c) and its IRT (d) composed by 36 HRRPs taken randomly from the frame in (a). bursts can properly represent the target, when the SNR on the data is sufficiently high. Nevertheless, the SNR on the final image is lower with respect to the signature obtained from the original frame, due to the incoherent integration of a smaller number of HRRPs.

This property of the algorithm is very important, since it is possible to apply the algorithm on a subset of the frame which covers the rotation period of the target, avoiding to use HRRPs affected by high level of noise or interference (e.g. jamming); on the other hand, it is possible to create simultaneously partial frames from different targets, jumping randomly from a target to another during the radar acquisitions in a multi-target scenario, computing a simultaneous classification of different objects.

For the analysis of target classification performance using partial data, the BSC model is taken into account. The training set for each class is the same described above. As in the previous analysis, even in this case the testing set in this analysis is realized considering noisy observations and continuously moving targets, with the SNR of data before the signature extraction processing varying within 0 and $10 \mathrm{~dB}$, and the rotation rate varying within $[0.25,3]$ Hz. Moreover, the RCS oscillations are represented by a lognormal distribution with unit mean and variance equal to 0.4 .

Figure 20 shows the performance in terms of $P_{S}, P_{M}$ and $P_{C}$, when $50 \%$ of the possible bursts are used, randomly taken from the entire HRRP frame within a rotation period of target. It is pointed out that in this case the number of bursts composing the frame varies with the rotation rate, as shown in Figure 14. Observing Figure 20a, Figure 20b and Figure 20c it is noted that the $P_{S}, P_{M}$ and $P_{C}$ do not change by using half of the available bursts instead of the entire frame (shown in Figure 16) when the rotation rate is smaller than 1.50 $\mathrm{Hz}$. For rotation rate greater than $1.50 \mathrm{~Hz}$ the algorithm performance is affected by using half of the HRRP frame, as consequence of significant decrement of $\widehat{M}$ for faster rotating targets. Specifically, for rotation rate of $3 \mathrm{~Hz}, P_{S}$ and $P_{M}$ are about 0.80 , while $P_{C}$ is about 0.70 . Finally, it is worth noting that the performance for each value of rotation rate does not change increasing the value of SNR from 0 to $10 \mathrm{~dB}$.

Figure 22 shows the performance in terms of $P_{S}, P_{M}$ and $P_{C}$, when 36 of the potential bursts are used, randomly taken from the entire HRRP frame within a rotation period of target. In this case, the percentage of bursts used for the algorithm varies on the angular rotation velocity, as shown in Figure 21. Moreover, the signal processing gain of the proposed algorithm is constant with respect to the target rotation rate.

It is worth noting that 36 bursts are sufficient to achieve 


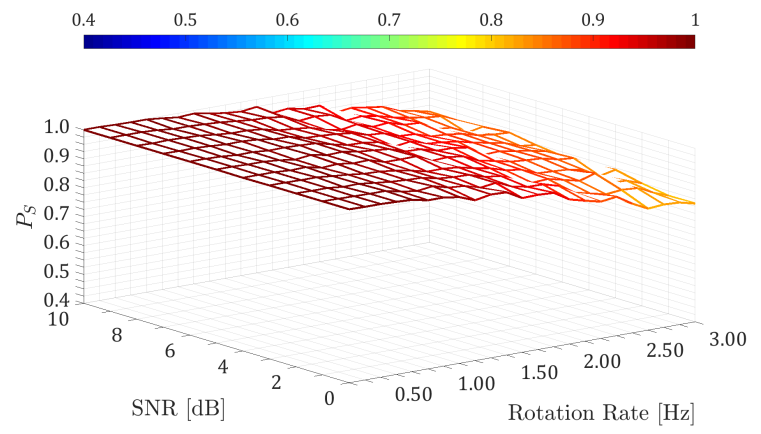

(a)

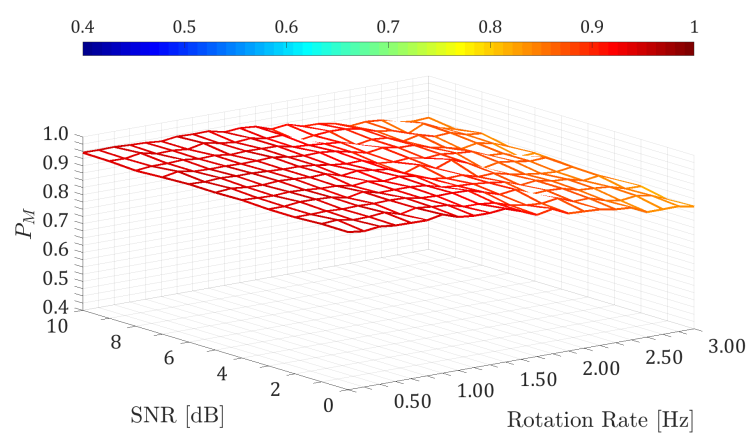

(b)

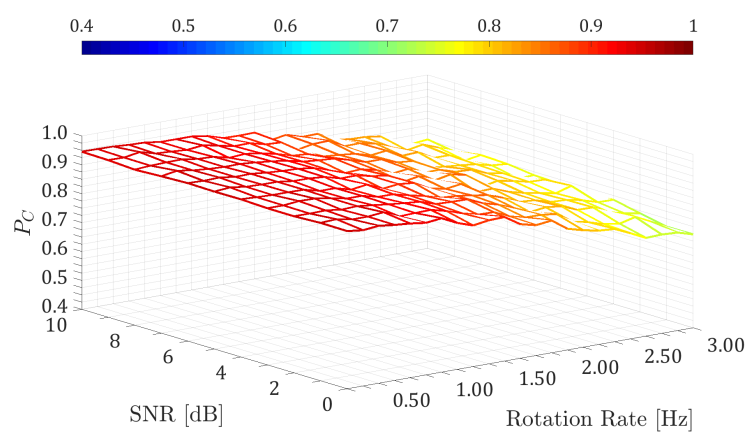

(c)

Figure 20: Performance in terms of $P_{S}$ (a), $P_{M}$ (b) and $P_{C}$ (c) by using $50 \%$ of the possible bursts randomly taken from the entire HRRP frame within a rotation period of target; the BSC model is used for the RCS.

$P_{S}$ and $P_{M}$ greater than 0.90 for almost all the considered rotation rate. In particular, it is pointed out that $P_{S}$ increases linearly with the rotation rate, going from about 0.90 when the rotation rate is $0.25 \mathrm{~Hz}$, up to about 0.95 when it is $3 \mathrm{~Hz}$, while the performance does not change for the considered values of SNR. This trend is due to completely random choice of bursts to synthesize the target signature. In fact, the entire HRRP frame for slower rotation rates contains a higher number of HRRPs, each of them corresponding to a different value

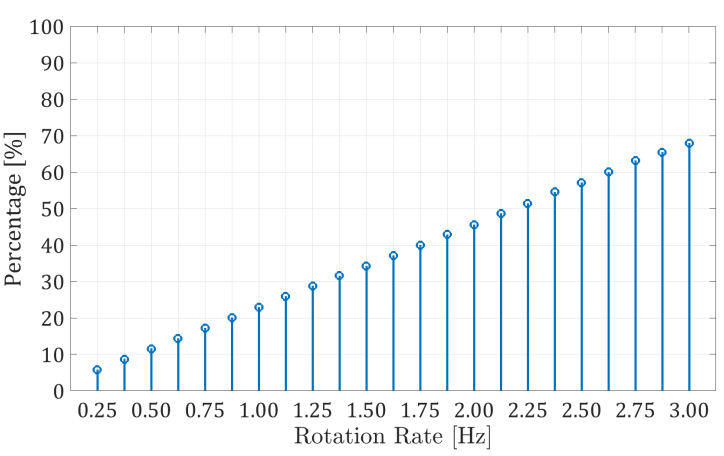

Figure 21: Partial HRRP frame dimension in percentage with respect to the total number of available HRRPs on varying the angular rotation velocity when 36 bursts are used for the classification algorithm.

of the aspect angle. Hence, some subsets of 36 bursts picked from the original frame may be concentrated in small regions of the frame, loosing information from a wider set of angles. On the other hand, for faster rotation rates the frame dimension decreases up to 52 bursts for rotation rate of $3 \mathrm{~Hz}$. Therefore, in this case it is easier that the 36 bursts cover the observation of rotation motion over better distributed angles, leading to better extraction of target signature. In the same way, from Figure 22b and Figure 22c it is observed that $P_{M}$ and $P_{C}$ increase linearly in $[0.88,0.925]$ and $[0.80,0.86]$, respectively, when the rotation rate increases from 0.25 $\mathrm{Hz}$ to $3 \mathrm{~Hz}$.

Therefore, it is possible to use pseudo-random burst repetition intervals to reconstruct properly the target signature for the presented algorithm, obtaining satisfactory classification performance. The number of bursts and the cadence with which they may be acquired depend on the rotation rate of the target, and have to be designed in order to observe the rotated target from a suitable set of angles.

\section{CONCLUSION}

In this paper a novel framework for the radar classification of BTs has been presented with the aim to distinguish between warheads and decoys. The presented algorithm employs the information relative to the range migrations of the principal target scatterers and the micro-motions, which are directly observable from a HRRP frame.

The effect of micro-motions on the SFWs radar return is analysed with emphases on the differences due to the signal polarization and due to the micro-motions exhibited by missile warheads and decoys. 


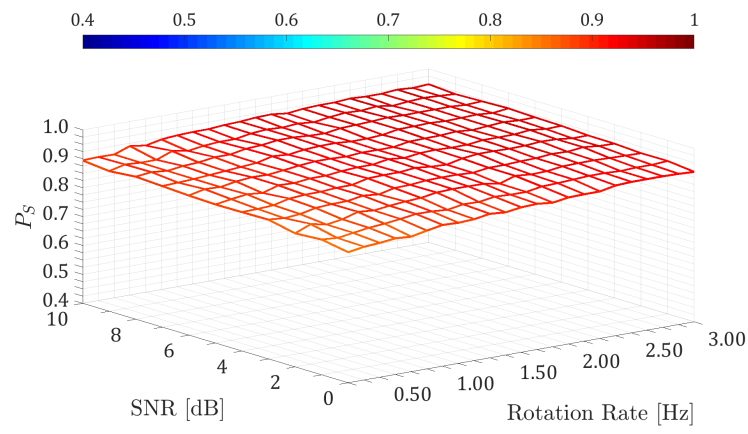

(a)

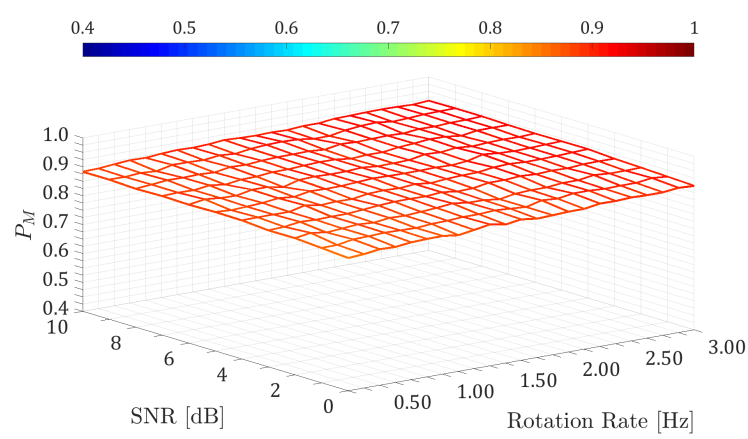

(b)

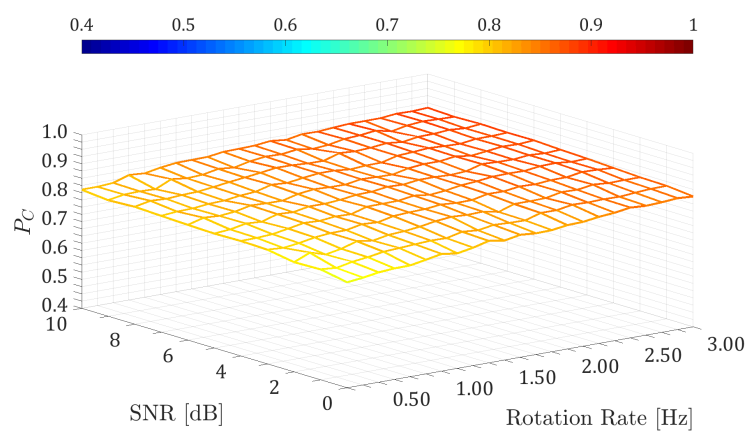

(c)

Figure 22: Performance in terms of $P_{S}$ (a), $P_{M}$ (b) and $P_{C}$ (c) by using 36 random bursts from the entire HRRP frame within a rotation period of target; the BSC model is used for the RCS.

The presented algorithm is based on the use of RT applied on the HRRP frame received from the target in order to extract a 2-D target signature. A feature vector for the final classification is evaluated by computing the pZ-moments from the 2-D target signature, guaranteeing classification being independent on the initial phase of the target micro-motions (no synchronization required).

The effectiveness of the proposed approach is tested on simulated SFWs radar data, obtained by considering three models for the RCS of the targets of interest:
BSC model, vertical and horizontal polarization models. The dataset for testing the algorithm has been realized for different values of the micro-motion parameters (e.g rotation velocities and precession angle), radar position angle and noise power.

The results have shown that the framework allows to discriminate between warheads and decoys with a satisfactory degree of correct shape and motion classification. In particular, the use of vertical polarization guarantees better performance than the horizontal polarization in terms of capability of shape identification and, consequently, of target classification. The reason is due to the higher scattering properties of points in proximity of the sharpest parts of the objects (e.g. cone tip) in the vertical polarization. The features are robust with respect to the SNR, the RCS oscillation and the HRRP distortions due to micro-movements. Specifically, this algorithm performs well in noise because the IRT has a high accumulation gain to sinusoidal curves in the target signature.

Lastly, the performance of the proposed classification algorithm was also evaluated in a random BRF scenario. Such target acquisition scenarios can occur in multitask systems where for example the radar would be able to switch between observing different targets in a pseudo-random manner. Simulation analysis showed that the algorithm is able to obtain satisfactory classification performance when the target is observed from a suitable set of angles.

The aspects of the designed radar waveform affects the target signature and the performance of the classification algorithm. In particular, the effect on the HRRP due to the target micro-motion velocity, in terms of radar range displacement from the real distance of the scattering point from the radar, depends on the number of subpulses used to synthesize the assigned total bandwidth and on the PRF. These parameters also have a significant impact on the final SNR of the target signature. Therefore, a further research on possible adaptable SFWs based on the estimated target micro-motion velocity could be conducted in the context of cognitive radar, improving the performance in presence of faster rotating object in lower SNR scenarios. Moreover, the design of a suitable model in agreement with to the target of interest (in terms of shape and dimension) and radar system parameters (e.g. polarization and bandwidth) can also lead to a model based classification algorithm guaranteeing high performance.

\section{APPENDIX}

In this appendix the expression of the complex coefficient for each scatterer is described for the two 
polarizations, vertical and horizontal, for the three shapes considered as target, namely cone, cylinder and cone plus cylinder. The details about the model design and validation are presented in [20].

\section{Cone}

For a conical target three principal scattering points are considering: the first is in correspondence of the cone tip, and other two are the intersection points between the circumference at cone bottom and the plane given by the symmetric axis and the LOS, as shown in Figure $4 a$.

Considering the cone semi-angle, $\gamma$, and the base radius, $r$ (see Figure $4 \mathrm{a}$ ), the modulus of scattering coefficients are expressed in (A.1), (A.2) and (A.3), where

$$
\mathcal{A}=\frac{\sin \left(\frac{\pi}{n_{2}}\right)}{n_{2}} \sqrt{\frac{r \csc (\alpha)}{k}}
$$

with

$$
\begin{aligned}
& n_{1}=2-\frac{2 \gamma}{\pi} \\
& n_{2}=n_{3}=\frac{3}{2}-\frac{\gamma}{\pi}
\end{aligned}
$$

and $k=\frac{2 \pi}{\lambda}$ the propagation factor, where $\lambda$ is the wavelength.

The phase of the coefficients are given by

$$
\begin{aligned}
& \rho_{1}=\frac{\pi}{4}-2 k\left(h_{1}+h_{2}\right) \cos (\alpha) \\
& \rho_{2}=\frac{\pi}{4}-2 k r \sin (\alpha) \\
& \rho_{3}=-\frac{\pi}{4}+2 k r \sin (\alpha)
\end{aligned}
$$

where $h_{1}$ and $h_{2}$ are the distance of the tip and the base centre with respect to the centre of mass, respectively. The choice of the sign in (A.2) and (A.3) depends on the polarization, specifically, the upper sign is associated to the vertical polarization for the incident electric field, while the lower to the horizontal polarization. Then, the scattered field from a conical target can be evaluated through (9) and (10).

The expressions of coefficients for $\alpha$ in proximity of values 0 and $\pi$ have been updated in [20] since singularities arise in (A.2) and (A.3). In particular in order to evaluate the total scattered field by a conical target for incidence at near tail-on, the polarization-independent contribution from (A.2) and (A.3) is substituted by

$$
\begin{gathered}
\left(\sigma_{2} e^{j \rho_{1}}+\sigma_{2} e^{j \rho_{3}}\right)_{p o l-i n d}= \\
2 \sqrt{\pi} k r^{2} \frac{J_{1}(2 k r \sin (\alpha))}{(2 k r \sin (\alpha))} e^{-j \frac{\pi}{2}}
\end{gathered}
$$

for $\alpha \in[0, \gamma]$, where $J_{1}(\cdot)$ is the Bessel function of first order. Defining $\alpha_{c a}$ as the axial crossover angle such that

$$
2 k r \sin \left(\alpha_{c a}\right)=2.44
$$

for $\alpha \in\left[\pi-\alpha_{c a}, \pi\right],(9)$ and (10) are substituted by

$$
\begin{aligned}
& \left(\sigma_{2} e^{j \rho_{2}}+\sigma_{3} e^{j \rho_{3}}\right) \\
& =\frac{2 r \sqrt{\pi} \sin \left(\frac{\pi}{n_{2}}\right)}{n_{2}} \times \\
& {\left[J_{0}(2 k r \sin (\alpha))\left\{\cos \left(\frac{\pi}{n_{2}}\right)-\cos \left(\frac{3 \pi}{n_{2}}\right)\right\}^{-1}\right.} \\
& -J_{1}(2 k r \sin (\alpha)) \frac{\frac{2 j \tan (\alpha)}{n_{2}} \sin \left(\frac{3 \pi}{n_{2}}\right)}{\left(\cos \left(\frac{\pi}{n_{2}}\right)-\cos \left(\frac{3 \pi}{n_{2}}\right)\right)^{2}} \\
& \left.\mp J_{2}(2 k r \sin (\alpha))\left\{\cos \left(\frac{\pi}{n_{2}}\right)-1\right\}^{-1}\right]
\end{aligned}
$$

where $J_{i}(\cdot)$, with $i=0,1,2$, is the Bessel function of $i$-th order. It is worth noting that (A.10) is independent on polarization.

\section{Cylinder}

For a cylindrical target, four principal scattering points, specifically two for each base taken by intersecting the circumferences at the bases and the plane given by the symmetric axis and the LOS, as shown in Figure $4 \mathrm{~b}$.

Due to the object symmetry along both the two axis of the cylinder (see Figure $4 b$ ), the expressions of the scattering coefficients are written for $\alpha \in\left[0, \frac{\pi}{2}\right]$. In particular, considering the axial crossover angle, $\alpha_{c a}$, and the broadside crossover angle, $\alpha_{c b}$, defined such that $[20]$

$$
\begin{aligned}
& 2 k r \sin \left(\alpha_{c a}\right)=2.44 \\
& 2 k h \cos \left(\alpha_{c b}\right)=2.25
\end{aligned}
$$

with $r$ the base radius and $h=h_{1}=h_{2}$ is the distance between the base centre and the phase reference centre, the modulus of the scattering coefficients for $\alpha \in] \alpha_{c a}, \frac{\pi}{2}-\alpha_{c b}[$ are expressed in (A.15), (A.16), (A.17) and (A.18), where

$$
\mathcal{B}=\frac{2}{3} \sin \left(\frac{2 \pi}{3}\right) \sqrt{\frac{r \csc (\alpha)}{k}}
$$

and $k$ is the propagation factor. Even for the cylinder coefficients the upper sign is associated to the vertical polarization and the lower to the horizontal polarization. 


$$
\begin{gathered}
\sigma_{1}=\left\{\begin{array}{lr}
\frac{\sin \left(\frac{\pi}{n_{1}}\right)}{4 k \sqrt{2 \pi} n_{1}} \sqrt{\frac{r \csc (\alpha)}{k}}\left[\left\{\cos \left(\frac{\pi}{n_{1}}\right)-\cos \left(\frac{2(\pi-\gamma-\alpha)}{n_{1}}\right)\right\}^{-1}\right] & \alpha<\pi-\gamma \\
0 & \pi-\gamma \leq \alpha \leq \pi
\end{array}\right. \\
\sigma_{2}=\mathcal{A}\left[\left\{\cos \left(\frac{\pi}{n_{2}}\right)-\cos \left(\frac{3 \pi-2 \alpha}{n_{2}}\right)\right\}^{-1} \mp\left\{\cos \left(\frac{\pi}{n_{2}}\right)-1\right\}^{-1}\right] \\
\sigma_{3}=\left\{\begin{array}{lr}
\mathcal{A}\left[\left\{\cos \left(\frac{\pi}{n_{3}}\right)-\cos \left(\frac{3 \pi+2 \alpha}{n_{3}}\right)\right\}^{-1} \mp\left\{\cos \left(\frac{\pi}{n_{3}}\right)-1\right\}^{-1}\right] & 0 \leq \alpha \leq \pi \\
0 & 0 \leq \alpha<\frac{\pi}{2}<\alpha \leq \pi \\
\gamma \leq \alpha \leq \frac{\pi}{2}
\end{array}\right.
\end{gathered}
$$

$$
\begin{aligned}
& \sigma_{1}=\mathcal{B}\left[\left\{\cos \left(\frac{2 \pi}{3}\right)-\cos \left(\frac{\pi+2 \alpha}{3 / 2}\right)\right\}^{-1} \mp\left\{\cos \left(\frac{2 \pi}{3}\right)-1\right\}^{-1}\right] \\
& \sigma_{2}=\mathcal{B}\left[\left\{\cos \left(\frac{2 \pi}{3}\right)-\cos \left(\frac{4 \alpha}{3}\right)\right\}^{-1} \mp\left\{\cos \left(\frac{2 \pi}{3}\right)-1\right\}^{-1}\right] \\
& \sigma_{3}=\mathcal{B}\left[\left\{\cos \left(\frac{2 \pi}{3}\right)-\cos \left(\frac{\pi-2 \alpha}{3 / 2}\right)\right\}^{-1} \mp\left\{\cos \left(\frac{2 \pi}{3}\right)-1\right\}^{-1}\right] \\
& \sigma_{4}=0
\end{aligned}
$$

The phase of the coefficients are given by

$$
\begin{aligned}
& \rho_{1}=\frac{\pi}{4}-2 k[r \sin (\alpha)+h \cos (\alpha)] \\
& \rho_{2}=\frac{\pi}{4}-2 k[r \sin (\alpha)-h \cos (\alpha)] \\
& \rho_{3}=-\frac{\pi}{4}+2 k[r \sin (\alpha)-h \cos (\alpha)] \\
& \rho_{4}=-\frac{\pi}{4}+2 k[r \sin (\alpha)+h \cos (\alpha)]
\end{aligned}
$$

For $\left.\alpha \in] 0, \alpha_{c a}\right]$ the polarization-independent contribution due to diffraction interjection between scatters $P_{1}$ and $P_{3}$ (see Figure $4 \mathrm{~b}$ ) is given by

$$
\begin{gathered}
\left(\sigma_{1} e^{j \rho_{1}}+\sigma_{3} e^{j \rho_{3}}\right)_{p o l-i n d}= \\
2 k r^{2} \sqrt{\pi} \frac{J_{1}(2 k r \sin (\alpha))}{2 k r \sin (\alpha)} e^{-j \frac{\pi}{2}-j 2 k h \cos (\alpha)}
\end{gathered}
$$

Then, in the evaluation of the scatter field, the polarization-independent contribution from (A.15) and (A.17) is substituted by (A.24). For LOS in the axial direction $(\alpha=0)$ the expression of the target RCS is

$$
\sigma(\alpha=0)=\frac{4 \pi a^{4}}{\lambda^{2}}
$$

while the phase is

$$
\rho(\alpha=0)=-\frac{\pi}{2}-2 k h
$$

Considering the interval $\alpha \in\left[\frac{\pi}{2}-\alpha_{c b}, \frac{\pi}{2}[\right.$, the polarization-independent contribution from (A.15) and
(A.16) is substituted by

$$
\begin{aligned}
& \left(\sigma_{1} e^{j \rho_{1}}+\sigma_{2} e^{j \rho_{2}}\right)_{p o l-i n d}= \\
& -2 h \sqrt{r k} \frac{\sin (2 k r \sin (\alpha))}{2 k r \sin (\alpha)} e^{j \frac{\pi}{4}-j 2 k r \sin (\alpha)}
\end{aligned}
$$

In the broadside direction $\left(\alpha=\frac{\pi}{2}\right)$ follows

$$
\begin{aligned}
& \sigma\left(\alpha=\frac{\pi}{2}\right)=k a(2 h)^{2} \\
& \rho\left(\alpha=\frac{\pi}{2}\right)=\frac{\pi}{4}-2 k r
\end{aligned}
$$

The scattered field from the cylinder for the other values of $\alpha$ can be evaluated thanks to the symmetry proprieties of the target.

\section{Cylinder plus cone}

Considering a target composed by a cone and a cylinder which share the base (see Figure 4c) the modulus of scattering coefficients are expressed in (A.30), (A.31), (A.32), (A.33) and (A.34), where, coherently to the other target shapes, the upper sign is associated to the vertical polarization and the lower to the horizontal polarization, and where

$$
\begin{aligned}
& \mathcal{C}_{1}=\frac{\sin \left(\frac{2 \pi}{n_{2}}\right)}{n_{2}} \sqrt{\frac{r \csc (\alpha)}{k}} \\
& \mathcal{C}_{2}=\frac{\sin \left(\frac{2 \pi}{n_{3}}\right)}{n_{3}} \sqrt{\frac{r \csc (\alpha)}{k}}
\end{aligned}
$$


with $r$ the base radius, and

$$
\begin{aligned}
& n_{1}=2-\frac{2 \gamma}{\pi} \\
& n_{2}=1+\frac{\gamma}{\pi} \\
& n_{3}=\frac{3}{2}
\end{aligned}
$$

The phase of the coefficients are given by

$$
\begin{aligned}
& \rho_{1}=\frac{\pi}{4}-2 k\left[r \sin (\alpha)+\left(h_{1}+\frac{h_{2}}{2}\right) \cos (\alpha)\right] \\
& \rho_{2}=\frac{\pi}{4}-2 k\left[r \sin (\alpha)+\frac{h_{2}}{2} \cos (\alpha)\right] \\
& \rho_{3}=\frac{\pi}{4}-2 k\left[r \sin (\alpha)-\frac{h_{2}}{2} \cos (\alpha)\right] \\
& \rho_{4}=-\frac{\pi}{4}+2 k\left[r \sin (\alpha)-\frac{h_{2}}{2} \cos (\alpha)\right] \\
& \rho_{5}=-\frac{\pi}{4}+2 k\left[r \sin (\alpha)+\frac{h_{2}}{2} \cos (\alpha)\right]
\end{aligned}
$$

considering that the phase reference centre is on the symmetric axis at the same distance from the cylinder bases centres.

As done for the conical target when incidence is at and near the nose-on axial aspect, even for target composed by a cone and a cylinder (A.31) and (A.33) for $0 \leq \alpha \leq$ $\gamma$ are substituted by

$$
\begin{aligned}
& \left(\sigma_{2} e^{j \rho_{2}}+\sigma_{4} e^{j \rho_{4}}\right)= \\
& \frac{2 r \sqrt{\pi} \sin \left(\frac{\pi}{n_{2}}\right)}{n_{2}} e^{-j 2 k h_{2} \cos (\alpha)} \times \\
& {\left[J_{0}(2 k r \sin (\alpha))\left\{\cos \left(\frac{\pi}{n_{2}}\right)-\cos \left(\frac{2 \pi}{n_{2}}\right)\right\}^{-1}\right.} \\
& -J_{1}(2 k r \sin (\alpha)) \frac{\frac{2 j \tan (\alpha)}{n_{2}} \sin \left(\frac{2 \pi}{n_{2}}\right)}{\left(\cos \left(\frac{\pi}{n_{2}}\right)-\cos \left(\frac{2 \pi}{n_{2}}\right)\right)^{2}} \\
& \left.\mp J_{2}(2 k r \sin (\alpha))\left\{\cos \left(\frac{\pi}{n_{2}}\right)-1\right\}^{-1}\right] \times
\end{aligned}
$$

Defining the cross over aspect angle $\alpha_{c a}$ as

$$
2 k r \sin \left(\alpha_{c a}\right)=2.44
$$

for $\pi-\alpha_{c a} \leq \alpha \leq \pi$, the polarization-independent contribution from (A.32) and (A.34) is substituted by

$$
\begin{gathered}
\left(\sigma_{3} e^{j \rho_{3}}+\sigma_{5} e^{j \rho_{5}}\right)_{p o l-i n d}= \\
2 \sqrt{\pi} k r^{2} \frac{J_{1}(2 k r \sin (\alpha))}{(2 k r \sin (\alpha))} e^{-j \frac{\pi}{2}+j 2 k h_{2} \cos (\alpha)}
\end{gathered}
$$

Finally, for the evaluation of scattered field in proximity of broadside direction, the polarization-independent contribution from (A.32) and (A.34) is substituted by

$$
\begin{aligned}
& \left(\sigma_{2} e^{j \rho_{2}}+\sigma_{3} e^{j \rho_{3}}\right)_{p o l-i n d}= \\
& -2 h_{2} \sqrt{r k} \frac{\sin \left(2 k h_{2} \cos (\alpha)\right)}{2 k h_{2} \cos (\alpha)} e^{j \frac{\pi}{4}-j 2 k r \sin (\alpha)}
\end{aligned}
$$

for $\alpha_{c b} \leq \alpha \leq \pi-\alpha_{c b}$, where the broadside cross over angle $\alpha_{c b}$ verify

$$
2 k h_{2} \cos \left(\alpha_{c b}\right)=2.25
$$

All other contributions to the total return from the target are well behaved in this angular region [20].

\section{ACKNOWLEDGMENT}

This work was supported by the Engineering and Physical Sciences Research Council (EPSRC) Grant number EP/K014307/1; and the MOD University Defence Research Collaboration (UDRC) in Signal Processing

\section{REFERENCES}

[1] A.M. Sessler, J.M. Cornwall, B. Dietz, S. Fetter, S. Frankel, R.L. Garwin, K. Gottfried, L. Gronlund, G.N. Lewis, T.A. Postol, and D.C. Wright, "Countermeasure: A technical evaluation of the operational effectiveness of the planned us national missile defense system," Tech. Rep., Union of Concerned Scientists MIT Security Studies Program, April 2000.

[2] Stephen D. Weiner and Sol M. Rocklin, "Discrimination performance requirements for ballistic missile defense," The Lincoln Laboratory Journal, vol. 7, no. 1, pp. 63-88, 1994.

[3] A. R. Persico, C. Clemente, D. Gaglione, C. V. Ilioudis, J. Cao, L. Pallotta, A. De Maio, I. Proudler, and J. J. Soraghan, "On model, algorithms, and experiment for micro-dopplerbased recognition of ballistic targets," IEEE Transactions on Aerospace and Electronic Systems, vol. 53, no. 3, pp. 10881108, June 2017.

[4] Isaac Bankman, Eric Rogala, and Richard Pavek, "Laser radar in ballistic missile defense," Johns Hopkins APL Technical Digest, vol. 22, no. 3, pp. 379-393, 2001.

[5] H. Gao, L. Xie, S. Wen, and Y. Kuang, "Micro-doppler signature extraction from ballistic target with micro-motions," IEEE Transactions on Aerospace and Electronic Systems, vol. 46, no. 4, pp. 1969-1982, Oct 2010.

[6] P. Lei, K. 1. Li, and Y. x. Liu, "Feature extraction and target recognition of missile targets based on micro-motion," in Signal Processing (ICSP), 2012 IEEE 11th International Conference on, Oct 2012, vol. 3, pp. 1914-1919.

[7] V.C. Chen, F. Li, S.S. Ho, and H. Wechsler, "Micro-Doppler effect in radar: Phenomenon, model, and simulation study," IEEE Transactions on Aerospace and Electronic Systems, vol. 42, no. 1, pp. 2-21, Jan 2006.

[8] M.E. Clark, "High range resolution techniques for ballistic missile targets," Tech. Rep., British Aerospace Land and Sea Systems, Newport Road, Cowes, Isle of Wight, PO3 1 8PF, United Kingdom, 1999 British Aerospace pic.

[9] Bo Liu and Wenge Chang, "Motion compensation for missileborne frequency stepped chirp radar," in IET International Radar Conference 2013, April 2013, pp. 1-6. 


$$
\begin{aligned}
& \sigma_{1}=\left\{\begin{array}{lr}
\frac{\sin \left(\frac{\pi}{n_{1}}\right)}{4 k \sqrt{2 \pi} n_{1}} \sqrt{\frac{r \csc (\alpha)}{k}}\left[\left\{\cos \left(\frac{\pi}{n_{1}}\right)-\cos \left(\frac{2(\pi-\gamma-\alpha)}{n_{1}}\right)\right\}^{-1}\right] \\
0 & \pi<\pi-\gamma \leq \alpha \leq \pi
\end{array}\right. \\
& \sigma_{2}=\left\{\begin{array}{lr}
\mathcal{C}_{1}\left[\left\{\cos \left(\frac{2 \pi}{3}\right)-\cos \left(\frac{\pi+2 \alpha}{3 / 2}\right)\right\}^{-1} \mp\left\{\cos \left(\frac{2 \pi}{3}\right)-1\right\}^{-1}\right] \\
0
\end{array}\right. \\
& \sigma_{3}=\left\{\begin{array}{lr}
\mathcal{C}_{2}\left[\left\{\cos \left(\frac{2 \pi}{3}\right)-\cos \left(\frac{4 \alpha}{3}\right)\right\}^{-1} \mp\left\{\cos \left(\frac{2 \pi}{3}\right)-1\right\}^{-1}\right] & 0<\alpha \leq \pi \\
0 & \alpha=0
\end{array}\right. \\
& \sigma_{4}=\left\{\begin{array}{lr}
\mathcal{C}_{1}\left[\left\{\cos \left(\frac{2 \pi}{3}\right)-\cos \left(\frac{\pi-2 \alpha}{3 / 2}\right)\right\}^{-1} \mp\left\{\cos \left(\frac{2 \pi}{3}\right)-1\right\}^{-1}\right] \\
0
\end{array}\right.
\end{aligned}
$$

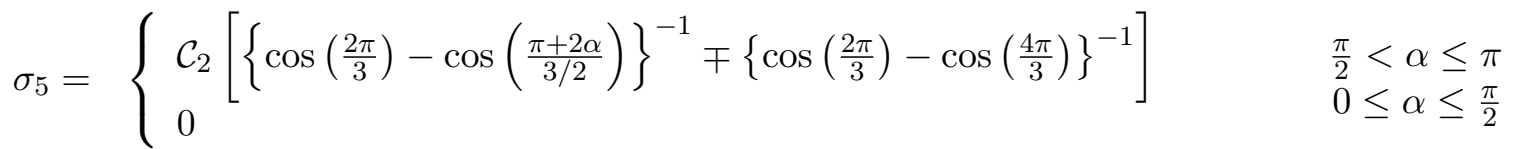

[10] S.R. Deans, The Radon Transform and Some of Its Applications, Dover Books on Mathematics Series. Dover Publications, 2007.

[11] J. C. Wood and D. T. Barry, "Radon transformation of time-frequency distributions for analysis of multicomponent signals," IEEE Transactions on Signal Processing, vol. 42, no. 11, pp. 3166-3177, Nov 1994.

[12] S. Stankovic, I. Djurovic, and I. Pitas, "Watermarking in the space/spatial-frequency domain using two-dimensional radonwigner distribution," IEEE Transactions on Image Processing, vol. 10, no. 4, pp. 650-658, Apr 2001.

[13] X. Bai, F. Zhou, M. Xing, and Z. Bao, "High resolution isar imaging of targets with rotating parts," IEEE Transactions on Aerospace and Electronic Systems, vol. 47, no. 4, pp. 25302543, OCTOBER 2011.

[14] T. Thayaparan, "Separation of target rigid body and microdoppler effects in sar/isar imaging," Technical memorandum, drdc ottawa tm 2006-187, Defence R\&D Canada, Ottawa, 2006.

[15] Y. Hua, J. Guo, and H. Zhao, "The usage of inverse-radon transformation in isar imaging," in 2014 IEEE International Conference on Control Science and Systems Engineering, Dec 2014, pp. 167-170.

[16] Li Kangle, Jiang Weidong, Liu Yongxiang, and Li Xiang, "Feature extraction of cone with precession based on microdoppler," in 2009 IET International Radar Conference, April 2009, pp. 1-5.

[17] A. R. Persico, C. Ilioudis, C. Clemente, and J. Soraghan, "Novel approach for ballistic targets classification from hrrp frame," in 2017 Sensor Signal Processing for Defence Conference (SSPD), Dec 2017, pp. 1-5.

[18] C. Clemente, L. Pallotta, I. Proudler, A. De Maio, J.J. Soraghan, and A. Farina, "Pseudo-zernike-based multi-pass automatic target recognition from multi-channel synthetic aperture radar," Radar, Sonar Navigation, IET, vol. 9, no. 4, pp. 457466, 2015.

[19] Zhang Qun, Luo Ying, and Chen Yong-an, Eds., Micro-
Doppler Characteristics of Radar Targets, ButterworthHeinemann, 2017.

[20] R.A. Ross, Investigation of Scattering Principles. Volume 3. Analytical Investigation, Defense Technical Information Center, 1969.

[21] A. De Maio, A. Farina, and G. Foglia, "Target fluctuation models and their application to radar performance prediction," IEE Proceedings - Radar, Sonar and Navigation, vol. 151, no. 5, pp. 261-269, Oct 2004.

[22] P. Swerling, "Radar probability of detection for some additional fluctuating target cases," IEEE Transactions on Aerospace and Electronic Systems, vol. 33, no. 2, pp. 698-709, April 1997.

[23] L. Liu, M. Ghogho, D. McLernon, and W. Hu, "Pseudo maximum likelihood estimations of ballistic missile precession frequency," in 2011 IEEE International Conference on Acoustics, Speech and Signal Processing (ICASSP), May 2011, pp. 3796-3799.

[24] L. Liu, M. Ghogho, D. McLernon, and W. Hu, "Ballistic missile precessing frequency extraction based on maximum likelihood estimation," in 2010 18th European Signal Processing Conference, Aug 2010, pp. 1562-1566.

[25] X. Bai and Z. Bao, "High-resolution 3d imaging of precession cone-shaped targets," IEEE Transactions on Antennas and Propagation, vol. 62, no. 8, pp. 4209-4219, Aug 2014.

[26] Honghua Yan, Xiongjun Fu, Xuehui Lei, Ping Li, and Meiguo Gao, "Parametric estimation of micro-doppler on spatial precession cone," in Proceedings of 2011 IEEE CIE International Conference on Radar, Oct 2011, vol. 1, pp. 613-616.

[27] G. Kertész, S. Szénási, and Z. Vámossy, "Application and properties of the radon transform for object image matching," in 2017 IEEE 15th International Symposium on Applied Machine Intelligence and Informatics (SAMI), Jan 2017, pp. 000353000358.

[28] AB Bhatia and E Wolf, "On the circle polynomials of Zernike and related orthogonal sets," in Mathematical Proceedings of the Cambridge Philosophical Society. Cambridge Univ Press, 1954, vol. 50, pp. 40-48. 
[29] He Sisan, Zhou Jianxiong, Zhao Hongzhong, and Le Daobin, "Estimating the precession angle of ballistic targets in midcourse based on hrrp sequence," in 2008 IEEE Radar Conference, May 2008, pp. 1-4.

[30] L. Li-hua, W. Zhuang, and H. Wei-dong, "Precession period extraction of ballistic missile based on radar measurement," in 2006 CIE International Conference on Radar, Oct 2006, pp. $1-4$.

[31] M. Daković and L. Stanković, "Estimation of sinusoidally modulated signal parameters based on the inverse radon transform," in 2013 8th International Symposium on Image and Signal Processing and Analysis (ISPA), Sept 2013, pp. 302307.

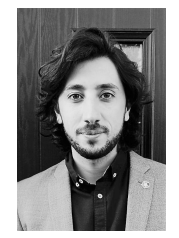

Adriano Rosario Persico (S'15) was born in Napoli, Italy, on October 7, 1988. $\mathrm{He}$ received his $\mathrm{BSc}$ and $\mathrm{MSc}$ from Università degli Studi di Napoli "Federico II", Italy, in 2011 and 2014, respectively, in Telecommunications Engineering. In 2018, he received the Ph.D. degree from the Department of Electronic and Electrical Engineering, University of Strathclyde, Glasgow, UK. Focus of his $\mathrm{PhD}$ research was on new advanced signal processing methods and algorithms for space situation awareness and defence against airborne threats.

Currently he is a Research Fellow with CNIT, viale G.P. Usberti, n. 181/A - 43124 Parma, c/o udr Università "Federico II", via Claudio 21, I-80125 Napoli, Italy, working on advanced radar signal processing algorithms for radar imaging by using passive bistatic radar and waveform design for cognitive radar in partnership with MBDA Italia, via Monte Flavio 45, Roma, Italy. His current research interests are in radar microDoppler, cognitive radar, compressed sensing, MIMO system and space based radar design for multi target detection, localisation and recognition for space situation awareness.

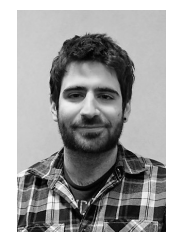

Christos V. Ilioudis (S'13) was born in Thessaloniki, Greece, on August 25, 1988. He received the Diploma degreefrom the Department of Informatics and Telecommunications Engineering, University Of Western Macedonia, Kozani, Greece, in 2012 and the M.Sc. degree with distinction in Electronics and Electrical Engineering from the University of Strathclyde, Glasgow, UK, in 2013. In 2017, he received his Ph.D. degree in the Department of Electronic and Electrical Engineering, University of Strathclyde, Glasgow, UK. Currently he is a Research Associate in the Department of Electronic and Electrical Engineering, University of Strathclyde, Glasgow, UK working on radar waveform design, passive radar and micro-Doppler.

His current research interests include joint radarcommunication systems, orthogonal chirp division multiplexing, UAV monitoring, micro-Doppler based human monitoring, and cognitive radar.

Dr. Ilioudis received the third position in the best student paper competition at IEEE International Radar Conference 2015, Arlington, USA. Also his paper was within the ten best papers (finalists) in the same competition at IEEE International Radar Conference 2016, Philadelphia, PA, USA.

Carmine Clemente (S'09-M'13-SM'18) received the Laurea cum laude (B.Sc.) and Laurea Specialistica cum laude (M.Sc.) degrees in telecommunications engineering from Università degli Studi del Sannio, Benevento, Italy, in 2006 and 2009, respectively. He received the Ph.D. degree from the Department of Electronic and Electrical Engineering, University of Strathclyde, Glasgow, UK, in 2012.

Currently, he is a Lecturer in the Department of Electronic and Electrical Engineering, University of Strathclyde, Glasgow, UK working on advanced Radar signal processing algorithm, MIMO radar systems, and micro-Doppler analysis. His research interests include synthetic aperture radar focusing and bistatic SAR focusing algorithms development, micro-Doppler signature analysis and extraction from multistatic radar platforms, micro-Doppler classification, and statistical signal processing.

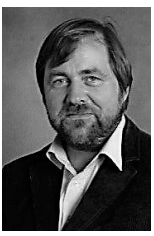

John J. Soraghan (S'83-M'84-SM'96) received the B.Eng. (Hons.) and M.Eng.Sc. degrees in electronic engineering from University College Dublin, Dublin, Ireland, in 1978 and 1983, respectively, and the $\mathrm{Ph} . \mathrm{D}$. degree in electronic engineering from the University of Southampton, Southampton, U.K., in 1989. His doctoral research focused on synthetic aperture radar processing on the distributed array processor.

After graduating, he worked with the Electricity Supply Board in Ireland and with Westinghouse Electric Corporation in the U.S. In 1986, he joined the Department of Electronic and Electrical Engineering, University of Strathclyde, Glasgow, U.K as a lecturer. He was a Manager of the Scottish Transputer Centre from 1988 to 1991, Manager with the DTI Parallel Signal Processing Centre from 1991 to 1995 and Head of the ICSP from 2005-2007. He became a Professor 
in Signal Processing in 2003 and has held the Texas Instruments Chair in Signal Processing since 2004. He is currently the Director of the Sensor Signal Processing Research Groups within the Centre for Signal and Image Processing (CeSIP) at Strathclyde. His main research interests are signal processing theories, algorithms, with applications to radar, sonar and acoustics, biomedical signal and image processing, video analytics, and condition monitoring.

Professor Soraghan has supervised 45 researchers to $\mathrm{PhD}$ graduation and has published over 340 technical publications. He is a member a Member of the IET and a Senior Member of the IEEE. 